\title{
Effective de Rham Cohomology - The General Case
}

\author{
Peter Scheiblechner \\ Lucerne University of Applied Sciences and Arts \\ School of Engineering and Architecture \\ 6048 Horw, Switzerland \\ peter.scheiblechner@hslu.ch
}

\begin{abstract}
Grothendieck has proved that each class in the de Rham cohomology of a smooth complex affine variety can be represented by a differential form with polynomial coefficients. We prove a single exponential bound on the degrees of these polynomials for varieties of arbitrary dimension. More precisely, we show that the $p$-th de Rham cohomology of a smooth affine variety of dimension $m$ and degree $D$ can be represented by differential forms of degree $(p D)^{\mathcal{O}(p m)}$. This result is relevant for the algorithmic computation of the cohomology, but is also motivated by questions in the theory of ordinary differential equations related to the infinitesimal Hilbert 16th problem.
\end{abstract}

Mathematics Subject Classification (2010): primary 14Q20, 14F40; secondary $68 \mathrm{~W} 30,34 \mathrm{C} 07$

Keywords: algebraic de Rham Cohomology, effective degree bound, differential forms, Gysin sequence

\section{Introduction}

Let $X$ be a smooth variety in $\mathbb{C}^{n}$. A fundamental result of Grothendieck says that the cohomology of $X$ can be described in terms of algebraic differential forms on $X$ [18]. More precisely, he proved that the singular cohomology of $X$ is isomorphic to the algebraic de Rham cohomology $H_{\mathrm{dR}}^{\bullet}(X)$, which is defined as the cohomology of the complex of algebraic differential forms on $X$. Hence, each cohomology class in $H_{\mathrm{dR}}^{p}(X)$ can be represented by a $p$-form

$$
\omega=\sum_{i_{1}<\cdots<i_{p}} \omega_{i_{1} \cdots i_{p}} \mathrm{~d} X_{i_{1}} \wedge \cdots \wedge \mathrm{d} X_{i_{p}}
$$

where the $\omega_{i_{1} \cdots i_{p}}$ are polynomial functions on $X$. However, Grothendieck's proof is not effective, i.e., it gives no information about the degrees of the 
polynomials $\omega_{i_{1} \cdots i_{p}}$, say in terms of defining equations for $X$. In [36] we proved a single exponential bound on their degrees in the case that $X$ is a hypersurface. In particular, if $X \subseteq \mathbb{C}^{n}$ is a smooth hypersurface of degree $D$, then each cohomology class in $H_{\mathrm{dR}}^{p}(X)$ can be represented by a differential form $\omega$ as in (1), where the $\omega_{i_{1} \cdots i_{p}}$ are polynomials of degree $D^{\mathcal{O}(p n)}$. The present paper generalizes this result to smooth varieties of arbitrary dimension.

\subsection{Motivation}

It is a long standing open question in algorithmic real algebraic geometry to find a single exponential time algorithm for computing the Betti numbers of a semialgebraic set. Single exponential time algorithms are known, e.g., for counting the connected components and computing the Euler characteristic of a semialgebraic set (for an overview see [2], for details and exhaustive bibliography see [3]). The best result in this direction states that for fixed $\ell$ one can compute the first $\ell$ Betti numbers of a semialgebraic set in single exponential time [1].

Over the complex numbers, one approach for computing Betti numbers is to compute the algebraic de Rham cohomology. In [30, 37] the de Rham cohomology of the complement of a complex affine variety is computed using Gröbner bases for $\mathcal{D}$-modules. This algorithm is extended in [38] to compute the cohomology of a projective variety. However, the complexity of these algorithms is not analyzed, and due to their use of Gröbner bases a good worst-case complexity is not to be expected. In [7] a single exponential time (in fact, parallel polynomial time) algorithm is given for counting the connected components, i.e., computing the zeroth de Rham cohomology, of a (possibly singular) complex variety. This algorithm is extended in [34] to one with the same asymptotic complexity for computing equations for the components. The first single exponential time algorithm for computing all Betti numbers of an interesting class of varieties is given in [35]. Namely, this paper shows how to compute the de Rham cohomology of a smooth projective variety in parallel polynomial time. In terms of structural complexity in the Turing model, these results are the best one can hope for, since the problem of computing a fixed Betti number (e.g., deciding connectedness) of a complex affine or projective variety defined over the integers is PSPACE-hard [33].

Besides being relevant for algorithms, our question also has connections to the theory of ordinary differential equations. The long standing infinitesimal Hilbert 16th problem has been solved in [5]. The authors derive a bound on the number of limit cycles generated from nonsingular energy level ovals (isolated periodic trajectories) in a non-conservative perturbation of a Hamiltonian polynomial vector field in the plane. It seems that their proof can be considerably generalized to solutions of certain linear systems of Pfaffian differential equations. Examples of such systems are provided by period matrices of polynomial maps, once the corresponding Gauss-Manin connexion can be explicitly constructed. For this construction one needs degree bounds for generators of the cohomology of the generic fibers of the polynomial map. 


\subsection{Known Cases}

We have shown in [7, Theorem 3.3] that the zeroth de Rham cohomology of $X$ is isomorphic to its zeroth singular cohomology even when $X$ is singular, and that this cohomology has a basis of degree $d^{\mathcal{O}\left(n^{2}\right)}$, if $X$ is defined by polynomials of degree $\leq d$.

It follows from the results of [35] that if $X$ has no singularities at infinity, i.e., the projective closure of $X$ in $\mathbb{P}^{n}$ is smooth, then each class in $H_{\mathrm{dR}}^{\bullet}(X)$ can be represented by a differential form of degree at most $m(\mathrm{em}+1) D$, where $m=\operatorname{dim} X, D=\operatorname{deg} X$, and $e$ is the maximal codimension of the irreducible components of $X$. However, in general $X$ does have singularities at infinity, and resolution of singularities has a very bad worst-case complexity [4].

Another special case with known degree bounds is the complement of a projective hypersurface, which we will actually use in this paper (see the proof of Theorem 7.1). The statement follows essentially from [9] and [10], the argument can be found in [12, Corollary 6.1.32], see also [11]. Let $f \in \mathbb{C}\left[X_{0}, \ldots, X_{n}\right]$ be a homogeneous polynomial, and consider $U:=\mathbb{P}^{n} \backslash \mathcal{Z}(f)$, which is an affine variety. Then, each class in $H_{\mathrm{dR}}^{p}(U)$ is represented by a (homogeneous) differential form

$$
\alpha / f^{p} \quad \text { with } \quad \operatorname{deg} \alpha=p \operatorname{deg} f
$$

(see $\S 2.1$ for the definition of the degree of a differential form). Since this result was already proved by Griffiths in a special case [16], we call it the GriffithsDeligne-Dimca (GDD) bound.

\subsection{Main Result}

The main result of this paper is that the algebraic de Rham cohomology of a smooth affine variety can be represented by differential forms of single exponential degree. More precisely, we prove the following.

Theorem 1.1. Let $k$ be an algebraically closed field of characteristic zero, and let $X$ be a smooth affine $m$-dimensional variety of degree $D$ over $k$. Then each cohomology class in $H_{\mathrm{dR}}^{p}(X)$ can be represented by a differential $p$-form of degree at most

$$
2^{2 p m+6 m+2} p^{2 p m+6 m+1} D^{4 p m+10 m+1}+D^{m+1}=(p D)^{\mathcal{O}(p m)} .
$$

Remark 1.2. (i) Note that we kept the additive term $D^{m+1}$, so that the bound is valid for $p=0$ as well (cf. Proposition 3.1).

(ii) If $X \subseteq \mathbb{A}^{n}$, then the term $D^{m+1}$ can be replaced by $(n+1) D^{2} / 4$ (Corollary 4.1).

(iii) For a hypersurface $X$, the slightly better bound of $D^{\mathcal{O}(p n)}$ is proved in [36]. 


\subsection{Proof Ideas}

The proof of Theorem 1.1 consists of two major steps. In a first step we reduce the question to the case of an irreducible locally closed hypersurface, and in a second step we prove a bound for this special case (Theorem 7.1).

The reduction to irreducible $X$ (Corollary 4.1) is an easy consequence of our characterization of the zeroth de Rham cohomology and the bound from [7]. In the present paper, using newer versions of the effective Nullstellensatz due to Kollár [26] and Jelonek [24], we improve this bound in two directions (Propositions 3.1 and 3.2). We also give an example showing that the second version is sharp up to a factor of $n+1$, where $n$ is the ambient dimension.

Then, if $X$ is irreducible of dimension $m$, a generic projection to a linear subspace of dimension $m+1$ restricts to a birational map from $X$ to a hypersurface $Y$. By Zariski's Main Theorem, there exist open dense subsets $U \subseteq X$ and $V \subseteq Y$, such that the projection maps $U$ isomorphically onto $V$. Using a geometric resolution of $X[14,31]$, we construct these locally closed sets effectively. More precisely, there exists a polynomial $f$ of degree $\leq D$ in $m+1$ variables, such that $U=X \backslash \mathcal{Z}(g)$ and $V=\mathcal{Z}(f) \backslash \mathcal{Z}(g)$, where $g$ is a partial derivative of $f$ (Lemma 4.2). It follows that a degree bound for $H_{\mathrm{dR}}^{\bullet}(V)$ implies a degree bound for $H_{\mathrm{dR}}^{\bullet}(U)$ (Corollary 4.4). Moreover, it is also not difficult to see that one can cover $X$ by such principal open subsets $U_{i}$ (Corollary 4.5). To finish the first major step, it remains to show how degree bounds for the open patches yield a bound for $H_{\mathrm{dR}}^{\bullet}(X)$ (Theorem 5.3). This is done using the concept of hypercohomology, which through a spectral sequence immediately yields an effective description of $H_{\mathrm{dR}}^{\bullet}(X)$ (Lemma 5.2) in terms of the total complex of the Cech-de Rham double complex. In order to derive a bound for the usual description in terms of global sections, we make the Čech cohomology effective (Lemma 5.1). In this construction we make essential use of Jelonek's effective Nullstellensatz [24].

The main idea for the treatment of the locally closed hypersurface $V$ is the same as in [36], namely to prove an effective version of the Gysin sequence (Theorem 6.1) and use the GDD bound for complements of hypersurfaces mentioned in $\$ 1.2$. However, in [36] we considered the case of a closed hypersurface $X \subseteq \mathbb{A}^{n}$, in which case the Gysin sequence yields an isomorphism $H_{\mathrm{dR}}^{p}\left(\mathbb{A}^{n} \backslash X\right) \stackrel{\sim}{\rightarrow} H_{\mathrm{dR}}^{p-1}(X)$, which we made effective. Here, we have only a locally closed subset $V=\mathcal{Z}(f) \backslash \mathcal{Z}(g)$ of $\mathbb{A}^{n}$. One idea is to treat $V$ as a closed subset of $\mathbb{A}^{n} \backslash \mathcal{Z}(g)$. Though we are able to construct an effective residue map in this setting, this map may not be surjective, since the surrounding space $\mathbb{A}^{n} \backslash \mathcal{Z}(g)$ has non-trivial cohomology in general. Our solution is to consider $V$ as a codimension 2 complete intersection $Z \subseteq \mathbb{A}^{n+1}$ through the isomorphism $\mathbb{A}^{n} \backslash \mathcal{Z}(g) \simeq \mathcal{Z}(g Y-1) \subseteq \mathbb{A}^{n+1}$. Since the Gysin sequence in the version of [19] does not hinge on codimension 1 , we get an isomorphism $H_{\mathrm{dR}}^{p+1}(W) \stackrel{\sim}{\rightarrow} H_{\mathrm{dR}}^{p-2}(Z)$, where $W:=\mathbb{A}^{n+1} \backslash Z$ (Corollary 6.2). Luckily, while the general complete intersection case seems considerably more difficult, we are able to prove the crucial Lemma (Lemma 6.4) exactly in this special case and obtain an effective Gysin sequence (Theorem 6.3). 
However, the price to pay is that now the complement $W$ is not affine anymore, so we need hypercohomology also to realize the de Rham cohomology of $W$, and a spectral sequence argument (Lemma 5.2) to apply the GDD bound (Lemma 5.4). Using local cohomology and another spectral sequence argument, we get a more succinct description of $H_{\mathrm{dR}}^{\bullet}(W)$ in terms of sheaf cohomology (Lemma 5.5).

\section{Preliminaries}

\subsection{Basic Notations and Facts}

Throughout this paper, let $k$ be an algebraically closed field of characteristic zero, and $R:=k\left[X_{1}, \ldots, X_{n}\right]$. An algebraic set or closed variety is the common zero set of polynomials $f_{1}, \ldots, f_{r} \in R$ in the affine space $\mathbb{A}^{n}=k^{n}$, i.e.,

$$
X=\mathcal{Z}\left(f_{1}, \ldots, f_{r}\right)=\left\{x \in k^{n} \mid f_{1}(x)=\cdots=f_{r}(x)=0\right\} .
$$

Note that $X$ may be reducible. More generally, the term variety will refer to a locally closed set, i.e., a Zariski open subset $X \subseteq Y$ of a closed variety $Y \subseteq \mathbb{A}^{n}$. A variety $X$ is called affine iff it is a principal open subset of $Y$, i.e.,

$$
X=Y \backslash \mathcal{Z}(g)=\mathcal{Z}\left(f_{1}, \ldots, f_{r}\right) \backslash \mathcal{Z}(g), \quad f_{1}, \ldots, f_{r}, g \in R .
$$

Indeed, these are exactly the varieties which are isomorphic to a closed variety, namely $\mathcal{Z}\left(f_{1}, \ldots, f_{r}, X_{n+1} g-1\right) \subseteq \mathbb{A}^{n+1}$. The (vanishing) ideal of an affine variety $X$ is defined as $I(X)=\{f \in R \mid f(x)=0 \forall x \in X\}$. By Hilbert's Nullstellensatz, $I(X)$ is the radical of the ideal $\left(f_{1}, \ldots, f_{r}\right):(g)$. The coordinate ring $A=k[X]$ of $X$ is the localization $(R / I(X))_{g}$. Such a ring $A$ will be called a (reduced) affine $k$-algebra.

In general, a variety is not affine. However, if $X=Y \backslash \mathcal{Z}\left(g_{1}, \ldots, g_{r}\right)$ with affine $Y$, then $X$ is covered by the affine open subsets $U_{i}:=X \backslash \mathcal{Z}\left(g_{i}\right)=Y \backslash \mathcal{Z}\left(g_{i}\right)$, $1 \leq i \leq r$.

The dimension $\operatorname{dim} X$ is the Krull dimension of $X \subseteq \mathbb{A}^{n}$ in the Zariski topology. In the case $\operatorname{dim} X=n-1$ we call $X$ a hypersurface. The degree $\operatorname{deg} X$ of an irreducible variety $X \subseteq \mathbb{A}^{n}$ of dimension $m$ is defined as the maximal cardinality of $X \cap L$ over all affine linear subspaces $L \subseteq \mathbb{A}^{n}$ of dimension $n-$ $m[29, \S 5 \mathrm{~A}]$. We define the (cumulative) degree $\operatorname{deg} X$ of a reducible variety $X$ to be the sum of the degrees of all irreducible components of $X$. It follows essentially from Bézout's Theorem that if $X \subseteq \mathbb{A}^{n}$ is closed and defined by polynomials of degree $\leq d$, then $\operatorname{deg} X \leq d^{n}[8]$.

An important ingredient of our work is the effective Nullstellensatz, which was first proved in $[6,25]$. A recent version of Jelonek [24] generalizes the statement to polynomials on general affine varieties.

Theorem 2.1. Let $X \subseteq \mathbb{A}^{n}$ be a closed subvariety of dimension $m$ and degree $D$, and let $g_{1}, \ldots, g_{t} \in R$ be polynomials of degree at most $d \geq 1$ without common 
zeros in $X$. Then there exist polynomials $h_{i} \in R$ with $1 \equiv \sum_{i} h_{i} g_{i} \bmod I(X)$ and

$$
\operatorname{deg}\left(h_{i} g_{i}\right) \leq \begin{cases}D d^{t} & \text { if } t \leq m, \\ D d^{m} & \text { if } t>m, d \geq 3, m \geq n-1, \\ 2 D d^{m}-1 & \text { else. }\end{cases}
$$

Proof. The first and the last case is due to [24]. The slightly better bound in the middle case follows from [25].

There is another version of the effective Nullstellensatz for arbitrary ideals due to Kollár [26]. We note a variant which follows easily from Theorem 6.2 in that paper.

Theorem 2.2. Let $X_{1}, \ldots, X_{t} \subseteq \mathbb{A}^{n}$ be closed varieties with $X_{1} \cap \cdots \cap X_{t}=\emptyset$. Then there exist $f_{i} \in I\left(X_{i}\right)$ such that

$$
\sum_{i} f_{i}=1 \quad \text { and } \quad \operatorname{deg} f_{i} \leq(n+1) \prod_{i} \operatorname{deg} X_{i} .
$$

\subsection{Completions}

In our proof we will also use the process of completion [13, Chapter 7]. Let $A$ be an affine algebra and $I$ an ideal in $A$. The completion $\widehat{A}=\widehat{A}_{I}$ of $A$ with respect to $I$ is defined as the inverse limit of the factor rings $A / I^{\nu}, \nu \geq 0$. There is a canonical map $A \rightarrow \widehat{A}$, whose kernel is $\bigcap_{\nu} I^{\nu}$, thus it is injective in our case. Alternatively, if $I=\left(f_{1}, \ldots, f_{r}\right)$, one can define $\widehat{A}$ as $A\left[\left[T_{1}, \ldots, T_{r}\right]\right] /\left(T_{1}-\right.$ $\left.f_{1}, \ldots, T_{r}-f_{r}\right)$, so its elements are power series in $f_{1}, \ldots, f_{r}$ [13, Exercise 7.11]. For instance, if $A=B[T]$ and $I=(T)$, then $\widehat{A}=B[[T]]$ is the ring of formal power series in $T$ with coefficients in $B$.

\subsection{Coherent Sheaves and Differential Forms}

Let $X$ be an affine variety. Then every $k[X]$-module $M$ gives rise to a sheaf $\widetilde{M}$ on $X$ such that, on a principal open subset $U=X \backslash \mathcal{Z}(g)$, the sections of $\widetilde{M}$ are given by $\Gamma(U, \widetilde{M})=M_{g}$, the localization of $M$ at $g$ [22, Proposition II.5.1]. Moreover, $\Gamma(U, \widetilde{M})$ is a $k[X]_{g}$-module which is compatible with restrictions. A sheaf $\mathcal{F}$ on $X$ is called coherent iff $\mathcal{F}=\widetilde{M}$ with a finitely generated $k[X]$ module $M$. An important example is the structure sheaf $\mathcal{O}_{X}=\widetilde{k[X]}$.

More generally, a sheaf $\mathcal{F}$ on a locally closed set $X$ is called coherent iff $X$ can be covered by affine open subsets $U_{i}$ such that all the restrictions $\mathcal{F} \mid U_{i}$ are coherent. In particular, if $\mathcal{F}$ is a coherent sheaf on $Y$ and $X \subseteq Y$ is an open subset, then $\mathcal{F} \mid X$ is a coherent sheaf on $X$.

Now let $A$ be a $k$-algebra (commutative, with 1 ). The module of Kähler differentials $\Omega_{A}:=\Omega_{A / k}$ is defined as the $A$-module generated by symbols $\mathrm{d} f$ for all $f \in A$, modulo the relations of Leibniz' rule and $k$-linearity of the universal derivation d: $A \rightarrow \Omega_{A}$. For instance, for the polynomial ring $R$, the module 
$\Omega_{R}$ is free with basis $\mathrm{d} X_{1}, \ldots, \mathrm{d} X_{n}$, and the universal derivation is given by $\mathrm{d} f=\sum_{i} \frac{\partial f}{\partial X_{i}} \mathrm{~d} X_{i}$. Now let $\Omega_{A}^{p}:=\bigwedge^{p} \Omega_{A}$ be the $p$-th exterior power of the $A$-module $\Omega_{A}$. We define the exterior differential d: $\Omega_{A}^{p} \rightarrow \Omega_{A}^{p+1}$ by setting $\mathrm{d}\left(f \mathrm{~d} g_{1} \wedge \cdots \wedge \mathrm{d} g_{p}\right):=\mathrm{d} f \wedge g_{1} \wedge \cdots \wedge \mathrm{d} g_{p}$ for all $f, g_{i} \in A$. It is easy to check that $\mathrm{d}$ satisfies the graded Leibniz' rule and $\mathrm{d} \circ \mathrm{d}=0$. This way we obtain the de Rham complex

$$
\Omega_{A}^{\bullet}: A=\Omega_{A}^{0} \stackrel{\mathrm{d}}{\longrightarrow} \Omega_{A}^{1} \stackrel{\mathrm{d}}{\longrightarrow} \cdots \stackrel{\mathrm{d}}{\longrightarrow} \Omega_{A}^{p} \longrightarrow \cdots
$$

of the algebra $A$.

Now let $Y$ be an affine variety with coordinate ring $A=k[Y]$. Then the sheaf of regular differential $p$-forms on $Y$ is defined as $\Omega_{Y}^{p}:=\widetilde{\Omega}_{A}^{p}$. The exterior differentials glue together to maps of sheaves $\mathrm{d}: \Omega_{Y}^{p} \rightarrow \Omega_{Y}^{p+1}$. More generally, if $X \subseteq Y$ is an open subset of an affine variety $Y$, then $\Omega_{X}^{p}:=\Omega_{Y}^{p} \mid X$. This way we obtain the (algebraic) de Rham complex

$$
\Omega_{X}^{\bullet}: \mathcal{O}_{X}=\Omega_{X}^{0} \stackrel{\mathrm{d}}{\longrightarrow} \Omega_{X}^{1} \stackrel{\mathrm{d}}{\longrightarrow} \cdots \stackrel{\mathrm{d}}{\longrightarrow} \Omega_{X}^{p} \longrightarrow \cdots
$$

of the variety $X$. We say that $X$ is smooth at $x \in X$ iff the stalk $\Omega_{X, x}$ is a free $\mathcal{O}_{X, x}$-module of rank $m$, where $m$ is the maximal dimension of all irreducible components of $X$ through $x$. In fact, in this case there is only one such component. We say that $X$ is smooth iff it is smooth at all its points. Note that in this case $\Omega_{X}^{p}=0$ for $p>m$.

The module of Kähler differentials of a complete ring may not be finitely generated (see, e.g., [13, Exercise 16.14]). In these cases we use the universally finite module of differentials, which is always finitely generated (see [27, §1112]). Let $A$ be an affine algebra, $I$ an ideal in $A$, and $\widehat{A}$ the completion of $A$ with respect to $I$. The completion of $\Omega_{A}$ with respect to $I$ is $\widehat{\Omega}_{\widehat{A}}=\widehat{A} \otimes_{A} \Omega_{A}$ and is called the universally finite module of differentials of $\widehat{A}$. There is a universally finite derivation $\mathrm{d}: \widehat{A} \rightarrow \widehat{\Omega}_{\widehat{A}}$ which is continuous, i.e., it commutes with infinite sums. For instance, for an affine algebra $B$ we have

$$
\widehat{\Omega}_{B[[T]]}=B[[T]] \otimes_{B[[T]]} \Omega_{B[T]}=B[[T]] \mathrm{d} T \oplus \Omega_{B},
$$

and the universally finite derivation is given by $\mathrm{d} f=\frac{\partial f}{\partial T} \mathrm{~d} T+\mathrm{d}_{B} f$ for $f \in B[[T]]$, where $\frac{\partial f}{\partial T}$ denotes the formal partial derivative with respect to $T$, and $\mathrm{d}_{B} f$ is coefficient-wise application of the differential of $B$ [27, Example 12.7].

\subsection{Sheaf and Local Cohomology}

Let $X$ be a variety. Formally, the $q$-th sheaf cohomology functor $H^{q}(X, \cdot)$ from the category of sheaves of abelian groups on $X$ to the category of abelian groups is defined as the $q$-th right derived functor of the global section functor $\Gamma(X, \cdot)$,

$$
H^{q}(X, \cdot)=R^{q} \Gamma(X, \cdot) .
$$


Since this definition is computationally quite inconvenient, we prefer a different description. Let $\mathcal{F}$ be a coherent sheaf and $\mathcal{U}:=\left\{U_{i} \mid 0 \leq i \leq t\right\}$ an affine open cover of $X$. The sheaf cohomology of $\mathcal{F}$ can be computed as the Cech cohomology with respect to $\mathcal{U}$, which is defined as follows. For a set of indices $0 \leq i_{0}, \ldots, i_{q} \leq t$ denote $U_{i_{0} \cdots i_{q}}:=U_{i_{0}} \cap \cdots \cap U_{i_{q}}$. We define the vector spaces

$$
C^{q}:=C^{q}(\mathcal{U}, \mathcal{F}):=\bigoplus_{0 \leq i_{0}<\cdots<i_{q} \leq t} \mathcal{F}\left(U_{i_{0} \cdots i_{q}}\right)
$$

and the linear maps $\delta^{q}: C^{q} \longrightarrow C^{q+1}$,

$$
\delta^{q}(\omega)_{i_{0} \cdots i_{q+1}}:=\sum_{\nu=0}^{q+1}(-1)^{\nu} \omega_{i_{0} \cdots \widehat{i_{\nu} \cdots i_{q+1}}} \mid U_{i_{0} \cdots i_{q+1}} .
$$

Then one checks that $\delta^{q+1} \circ \delta^{q}=0$ for all $q \geq 0$, so that $\left(C^{\bullet}(\mathcal{U}, \mathcal{F}), \delta^{\bullet}\right)$ is a complex called the $\check{C} e c h$ complex. Its cohomology defines the cohomology $H^{\bullet}(\mathcal{U}, \mathcal{F})$, i.e.,

$$
H^{q}(\mathcal{U}, \mathcal{F}):=\frac{\operatorname{ker} \delta^{q}}{\operatorname{im} \delta^{q-1}}, \quad q \geq 0
$$

By [22, Theorem III.4.5] there is a natural isomorphism

$$
H^{q}(X, \mathcal{F}) \simeq H^{q}(\mathcal{U}, \mathcal{F}) \text { for all } \quad q \geq 0 .
$$

Generally, $H^{0}(X, \mathcal{F})$ is isomorphic to the space of global sections $\Gamma(X, \mathcal{F})$. Moreover, if $X$ is affine, the higher cohomology of $\mathcal{F}$ vanishes, i.e.,

$$
H^{q}(X, \mathcal{F})=0 \text { for } q>0 .
$$

A relative variant of sheaf cohomology is local cohomology. Let $X$ be a variety and $Y \subseteq X$ a closed subset of $X$. For a sheaf of abelian groups $\mathcal{F}$ on $X$ let $\Gamma_{Y}(X, \mathcal{F})$ be the subgroup of $\Gamma(X, \mathcal{F})$ consisting of all sections $s$ whose support is in $Y$, i.e.,

$$
\left\{s_{x} \neq 0\right\} \subseteq Y,
$$

where $s_{x}$ denotes the germ of $s$ in the stalk $\mathcal{F}_{x}$. The $q$-th local cohomology functor with supports in $Y$ from the category of sheaves of abelian groups on $X$ to the category of abelian groups is defined as the $q$-th right derived functor of the functor $\Gamma_{Y}(X, \cdot)$,

$$
H_{Y}^{q}(X, \cdot)=R^{q} \Gamma_{Y}(X, \cdot) .
$$

Note that, if $X$ is irreducible and $Y \neq X$, then $\Gamma_{Y}(X, \mathcal{F})=0$ for all coherent sheaves $\mathcal{F}$ on $X$. Nevertheless, the definition gives non-trivial local cohomology groups also in this case! There is an explicit description of local cohomology in terms of Koszul cohomology, which can also be interpreted as Čech cohomology [19, Theorem 2.3]. We will use this interpretation in a special case in the proof of Lemma 5.5, see (13). What makes local cohomology particularly useful, is the following long exact sequence [19, Corollary 1.9]. Let $Y \subseteq X$ be closed and $U:=X \backslash Y$. Then there is an exact sequence

$$
\cdots \rightarrow H^{q-1}(X, \mathcal{F}) \rightarrow H^{q-1}(U, \mathcal{F} \mid U) \rightarrow H_{Y}^{q}(X, \mathcal{F}) \rightarrow H^{q}(X, \mathcal{F}) \rightarrow \cdots .
$$




\subsection{Hypercohomology and de Rham Cohomology}

Let $X$ be a variety and consider a complex of coherent sheaves $\left(\mathcal{F}^{\bullet}, \mathrm{d}\right)$ on $X$ with $\mathcal{F}^{p}=0$ for $p<0$. Then, for an affine open cover $\mathcal{U}$, the Cech complexes $C^{\bullet}\left(\mathcal{U}, \mathcal{F}^{p}\right)$ as defined in $\S 2.4$ fit together to the Čech double complex $C^{\bullet \bullet \bullet}:=$ $C^{\bullet}, \bullet\left(\mathcal{U}, \mathcal{F}^{\bullet}\right)$ by setting

$$
C^{p, q}\left(\mathcal{U}, \mathcal{F}^{\bullet}\right)=\bigoplus_{i_{0}<\cdots<i_{q}} \mathcal{F}^{p}\left(U_{i_{0} \cdots i_{q}}\right) \quad \text { for all } \quad p, q \geq 0 .
$$

The two differentials are the one induced by the differential $\mathrm{d}$ of $\mathcal{F}$ and the Čech differential $\delta^{\bullet}$ defined by (2). Define the total complex of $C^{\bullet} \bullet \bullet$ by

$$
\operatorname{tot}^{\ell}\left(C^{\bullet \bullet \bullet}\right):=\bigoplus_{p+q=\ell} C^{p, q}, \quad \mathrm{~d}^{\text {tot }}:=\delta^{q}+(-1)^{q} \mathrm{~d} \text { on } C^{p, q} .
$$

Since the two differentials commute, one easily checks that $\mathrm{d}^{\text {tot }} \circ \mathrm{d}^{\text {tot }}=0$. The hypercohomology of the complex of sheaves $\mathcal{F}^{\bullet}$ is defined as the cohomology of the total complex

$$
\mathbb{H}^{\ell}\left(X, \mathcal{F}^{\bullet}\right):=H^{\ell}\left(\operatorname{tot}^{\bullet}\left(C^{\bullet \bullet \bullet}\right)\right) \quad \text { for } \quad \ell \geq 0 .
$$

As for any double complex $[28, \S 2.4]$, there are two spectral sequences

$$
\begin{aligned}
& { }_{I} E_{2}^{p, q}=H^{p}\left(H^{q}\left(X, \mathcal{F}^{\bullet}\right), \mathrm{d}\right) \quad \Rightarrow \quad \mathbb{H}^{p+q}\left(X, \mathcal{F}^{\bullet}\right) \quad \text { and } \\
& { }_{I I} E_{2}^{p, q}=H^{q}\left(H^{p}\left(C^{\bullet \bullet \bullet}, \mathrm{d}\right), \delta^{\bullet}\right) \quad \Rightarrow \quad \mathbb{H}^{p+q}\left(X, \mathcal{F}^{\bullet}\right) \text {. }
\end{aligned}
$$

If $X$ is affine, then $H^{q}\left(X, \mathcal{F}^{\bullet}\right)=0$ for all $q>0$. Consequently, the first spectral sequence implies that $\mathbb{H}^{\bullet}\left(X, \mathcal{F}^{\bullet}\right) \simeq H^{\bullet}\left(\Gamma\left(X, \mathcal{F}^{\bullet}\right)\right)$.

The algebraic de Rham cohomology of a variety $X$ is defined as the hypercohomology of the algebraic de Rham complex

$$
H_{\mathrm{dR}}^{\bullet}(X):=\mathbb{H}^{\bullet}\left(X, \Omega_{X}^{\bullet}\right) .
$$

The corresponding double complex is called Čech-de Rham double complex. In particular, if $X$ is affine, then we have

$$
H_{\mathrm{dR}}^{\bullet}(X)=H^{\bullet}\left(\Omega_{A}^{\bullet}\right), \quad \text { where } \quad A=k[X] .
$$

In general, since the $U_{i_{0} \cdots i_{q}}$ are affine, the last equation implies that the first term of the second spectral sequence of the Čech-de Rham double complex is

$$
{ }_{I I} E_{1}^{p, q}=\bigoplus_{i_{0}<\cdots<i_{q}} H_{\mathrm{dR}}^{p}\left(U_{i_{0} \cdots i_{q}}\right) \quad \Rightarrow \quad H_{\mathrm{dR}}^{p+q}(X),
$$

even when $X$ is not affine.

Fundamental for us is the result of [18] stating that if $k=\mathbb{C}$, then the de Rham cohomology $H_{\mathrm{dR}}^{\bullet}(X)$ of a smooth variety $X$ is naturally isomorphic to the singular cohomology of $X$. 


\section{$2.6 \quad$ Filtrations}

We are mainly interested in degree bounds for the de Rham cohomology a closed variety $X \subseteq \mathbb{A}^{n}$, but in the course of the proof we also work with principal open subsets of the form $U=X \backslash \mathcal{Z}(g)$. The regular functions on $U$ have a power of $g$ as denominator, so also their order becomes important. To define the notions of degree and order in a precise and convenient way, we use the language of (double-)filtrations.

Let $A=R / I$ be the coordinate ring of a closed variety $X \subseteq \mathbb{A}^{n}$. For $f \in R$ we denote by $\bar{f}$ its residue class in $A$. We set

$$
\operatorname{deg} \bar{f}:=\min \{\operatorname{deg} h \mid h \in R, \bar{h}=\bar{f}\},
$$

where $\operatorname{deg} h$ denotes the total degree of $h$. We have $\operatorname{deg}(\bar{f} \bar{g}) \leq \operatorname{deg} \bar{f}+\operatorname{deg} \bar{g}$, but note that this inequality may be strict. We have the filtration by degree

$$
k=D^{0} A \subseteq \cdots \subseteq D^{d} A \subseteq D^{d+1} A \subseteq \cdots \subseteq A
$$

on $A$ given by

$$
D^{d} A:=\{f \in A \mid \operatorname{deg} f \leq d\},
$$

which satisfies

$$
D^{d} A \cdot D^{e} A \subseteq D^{d+e} A \text { for all } d, e \in \mathbb{Z} .
$$

We also consider modules $M$ over $A$ equipped with a filtration

$$
\cdots \subseteq F^{d} M \subseteq F^{d+1} M \subseteq \cdots \subseteq M
$$

which we assume to be compatible with $D^{\bullet} A$, i.e., $D^{d} A \cdot F^{e} M \subseteq F^{d+e} M$ for all $d, e \in \mathbb{Z}$. Given such a filtraton, one can define a degree by setting

$$
\operatorname{deg} x:=\min \left\{d \mid x \in F^{d} M\right\} \in \mathbb{Z} \quad \text { for } \quad x \in M .
$$

For a complex of $A$-modules $C^{\bullet}$, all equipped with a filtration $F^{\bullet} C^{\bullet}$, we define the induced filtration on the cohomology $H^{p}\left(C^{\bullet}\right)$ by setting

$$
F^{d} H^{p}\left(C^{\bullet}\right):=\operatorname{im}\left(F^{d} C^{p} \cap \operatorname{kerd} \rightarrow H^{p}\left(C^{\bullet}\right)\right),
$$

where d: $C^{p} \rightarrow C^{p+1}$ denotes the differential of the complex. For a filtered $k$-vector space $F^{\bullet} M$ we define

$$
\operatorname{deg}(M):=\inf \left\{d \in \mathbb{Z} \mid F^{d} M=M\right\} .
$$

Note that $\operatorname{deg}(M)=\infty$ if no such $d$ exists.

Now let $g \in R$ be a non-zerodivisor on $A$ and consider the localization $A_{g}$. The order of $h \in A_{g}$ with respect to $g$ is defined by

$$
\operatorname{ord}_{g} h:=\min \left\{s \in \mathbb{N} \mid \exists f \in A: h=\frac{f}{g^{s}}\right\} .
$$


Sometimes we drop the index $g$, if it is clear from the context. We have the filtration by order

$$
A=P^{0} A_{g} \subseteq \cdots \subseteq P^{s} A_{g} \subseteq P^{s+1} A_{g} \subseteq \cdots \subseteq A_{g},
$$

where

$$
P^{s} A_{g}:=\frac{1}{g^{s}} A=\left\{h \in A_{g} \mid \operatorname{ord}_{g} h \leq s\right\} .
$$

The definition of a convenient notion of degree in $A_{g}$ is problematic. We illustrate this in an

Example 2.3. Let $A=k\left[X_{1}, X_{2}, X_{3}\right] /\left(X_{1}^{d} X_{2}-X_{3}\right), g=X_{2}$, and consider the element

$$
a:=\overline{X_{3}} / \bar{g} \in A_{g} .
$$

When we account for the denominator as negative degree, then $a$ should have degree zero. On the other hand we have

$$
a={\overline{X_{1}}}^{d} \in A_{g},
$$

which is of degree $d$. We see that the degree depends on the representation of the element. Furthermore, in this example, decreasing the order by 1 increases the degree of the numerator arbitrarily.

However, on a localization $R_{g}$ of the polynomial ring, we define the degree of $h \in R_{g}$ by

$$
\operatorname{deg} h:=\operatorname{deg} f-s \operatorname{deg} g, \quad \text { where } \quad h=\frac{f}{g^{s}}, \quad f \in R, \quad s \in \mathbb{N} .
$$

Note that the degree can be arbitrarily small, so it induces an unbounded filtration by degree $D^{\bullet} R_{g}$. In $R_{g}$ we define the double filtration $F^{\bullet \bullet} \bullet R_{g}$ by

$$
F^{s, d} R_{g}:=\left\{h \in R_{g} \mid \operatorname{ord}_{g} h \leq s, \operatorname{deg} h \leq d\right\}
$$

Now, on $A_{g}$ we define

$$
F^{s, d} A_{g}:=\pi\left(F^{s, d} R_{g}\right)
$$

where $\pi: R_{g} \rightarrow A_{g}$ is the natural projection. Note that this double filtration depends on the representative $g \in R$ of $\bar{g} \in A$. In our applications, we will always consider a fixed representative. We have

$$
F^{s, d} A_{g} \subseteq F^{s+1, d} A_{g} \cap F^{s, d+1} A_{g} \quad \text { and } \quad F^{s, d} A_{g} \cdot F^{s^{\prime}, d^{\prime}} A_{g} \subseteq F^{s+s^{\prime}, d+d^{\prime}} A_{g} .
$$

Now let $M$ be an $A_{g}$-module equipped with a double filtration $F^{\bullet \bullet \bullet} M$, i.e., vector subspaces $F^{s, d} M \subseteq M$ for $s, d \in \mathbb{Z}$ such that $F^{s, d} M \subseteq F^{s+1, d} M \cap$ $F^{s, d+1} M$ for all $s, d \in \mathbb{Z}$. We assume that it is compatible with the filtration $F^{\bullet \bullet \bullet} A_{g}$, i.e.,

$$
F^{s, d} A_{g} \cdot F^{s^{\prime}, d^{\prime}} M \subseteq F^{s+s^{\prime}, d+d^{\prime}} M \quad \text { for all } \quad s, s^{\prime}, d, d^{\prime} \in \mathbb{Z} .
$$


Then we define the set

$$
B\left(F^{\bullet, \bullet} M\right):=\left\{(s, d) \in \mathbb{Z}^{2} \mid F^{s, d} M=M\right\} .
$$

If the double filtration is understood from the context, we also simply write $B(M)$. Note that with $(s, d) \in B\left(F^{\bullet, \bullet} M\right)$ we have $(s, d)+\mathbb{N}^{2} \subseteq B\left(F^{\bullet} \bullet M\right)$. A natural example is the localization $M=N_{g}$ of an $A$-module $N$. If $N$ has a compatible filtration $F^{\bullet} N$, then $M$ carries a compatible double filtration $F^{\bullet}, \bullet^{\prime} M$ by setting

$$
F^{s, d} M:=\frac{1}{g^{s}} F^{d+s \operatorname{deg} g} N .
$$

Similarly as in (3), a double filtration on a complex induces a double filtration on its cohomology. A double filtration on a double complex induces in a natural way a double filtation on its total complex.

Particularly important for us are the modules of Kähler differential forms. The $A_{g}$-module $\Omega_{A_{g}}^{p}$ is the localization $\Omega_{A_{g}}^{p}=\left(\Omega_{A}^{p}\right)_{g}$ of the $A$-module $\Omega_{A}^{p}[13$, Proposition 16.9]. We first define the filtration by degree on $\Omega_{A}^{p}$ by

$$
D^{d} \Omega_{A}^{p}:=\left\{\sum_{i_{1}<\cdots<i_{p}} f_{i_{1} \cdots i_{p}} \mathrm{~d} X_{i_{1}} \wedge \cdots \wedge \mathrm{d} X_{i_{p}} \mid f_{i_{1} \cdots i_{p}} \in D^{d-p} A\right\}
$$

and note that it is compatible with the filtration $D^{\bullet} A$. We account for the differentials in this definition of the degree, so that the differential map has degree zero, i.e.,

$$
\mathrm{d}\left(D^{d} \Omega_{A}^{p}\right) \subseteq D^{d} \Omega_{A}^{p+1} .
$$

We have an induced degree function satisfying

$$
\operatorname{deg} f \mathrm{~d} X_{i_{1}} \wedge \cdots \wedge \mathrm{d} X_{i_{p}}=\operatorname{deg} f+p \quad \text { for } \quad f \in A .
$$

Now, according to (6), the filtration $D^{\bullet} \Omega_{A}^{p}$ induces a compatible double filtration on the localization $\Omega_{A_{g}}^{p}$. We have

$$
\begin{aligned}
F^{s, d} \Omega_{A_{g}}^{p} & =\frac{1}{g^{s}} D^{d+s \operatorname{deg} g} \Omega_{A}^{p} \\
& =\left\{\sum_{i_{1}<\cdots<i_{p}} f_{i_{1} \cdots i_{p}} \mathrm{~d} X_{i_{1}} \wedge \cdots \wedge \mathrm{d} X_{i_{p}} \mid f_{i_{1} \cdots i_{p}} \in F^{s, d-p} A_{g}\right\},
\end{aligned}
$$

and call it the standard double filtration. We also have an order function on $\Omega_{A_{g}}^{p}$ satisfying

$$
\operatorname{ord}_{g} f \mathrm{~d} X_{i_{1}} \wedge \cdots \wedge \mathrm{d} X_{i_{p}}=\operatorname{ord}_{g} f \quad \text { for } \quad f \in A_{g} .
$$

With these definitions, the differential d: $\Omega_{A_{g}}^{p} \rightarrow \Omega_{A_{g}}^{p+1}$ satisfies

$$
\mathrm{d}\left(F^{s, d} \Omega_{A_{g}}^{p}\right) \subseteq F^{s+1, d} \Omega_{A_{g}}^{p+1} .
$$

Using these notations and conventions, we formulate an affine version of the GDD bound. 
Lemma 2.4. Let $f \in R$ and $U:=\mathbb{A}^{n} \backslash \mathcal{Z}(f)$. Then, with respect to the standard double filtration, we have

$$
p \cdot(1,1) \in B\left(H_{\mathrm{dR}}^{p}(U)\right) .
$$

Proof. Denote by $\tilde{f}$ the generous homogenization $X_{0}^{d+1} f\left(X / X_{0}\right)$, where $d:=$ $\operatorname{deg} f$. Then we have $U=\mathbb{P}^{n} \backslash \mathcal{Z}(\widetilde{f})$. As stated in $\S 1.2$, each cohomology class in $H_{\mathrm{dR}}^{p}(U)$ is represented by a differential form $\widetilde{\alpha} / \widetilde{f}^{p}$, where $\widetilde{\alpha}$ is a homogeneous $p$-form on $\mathbb{A}^{n+1}$ of degree $\operatorname{deg} \widetilde{\alpha}=p(d+1)$. Dehomogenizing yields a form $\omega=\alpha / f^{p}$ with $\operatorname{deg} \alpha \leq p(d+1)$, hence $\omega \in F^{p, p} \Omega_{R_{f}}^{p}$.

\section{Zeroth Cohomology}

In this section we discuss old and new results about the zeroth de Rham cohomology of a closed variety $X \subseteq \mathbb{A}^{n}$. This is a somewhat special case, since $H_{\mathrm{dR}}^{0}(X)$ characterizes the connected components of $X$ even if $X$ is singular.

We fix the notation for this section. Let $X=Z_{1} \cup \cdots \cup Z_{t}$ be the decomposition of $X$ into connected components, set $D:=\operatorname{deg} X$ and $D_{i}:=\operatorname{deg} Z_{i}$. By the results of $[7, \S 3.1 .2]$, there is a direct product decomposition of the coordinate ring

$$
k[X] \simeq \prod_{i=1}^{t} k\left[Z_{i}\right]
$$

which corresponds to a maximal complete set of pairwise orthogonal idempotents $e_{1}, \ldots, e_{t} \in k[X]$. Here, $e_{i}$ is the function which is equal to 1 on $Z_{i}$ and vanishes on $X \backslash Z_{i}$. Furthermore, we have proved in [7] that $e_{1}, \ldots, e_{t}$ is a basis of $H_{\mathrm{dR}}^{0}(X)$ and that $\operatorname{deg} e_{i} \leq d^{\mathcal{O}\left(n^{2}\right)}$, if $X$ is given by polynomials of degree $\leq d$. Due to Jelonek's version of the effective Nullstellensatz, we are able to improve this bound.

Proposition 3.1. Let $X \subseteq \mathbb{A}^{n}$ be a closed variety of dimension $m$ and degree $D$. Then $X$ has $\operatorname{dim} H_{\mathrm{dR}}^{0}(X)$ connected components, and

$$
\operatorname{deg}\left(H_{\mathrm{dR}}^{0}(X)\right) \leq D^{m+1} .
$$

Proof. The result is known for $m=0$, so assume $m \geq 1$. We construct the idempotents $e_{i}$ as follows. According to [7, Proposition 2.1], each $Z_{i}$ can be defined by (many) polynomials $f_{i \nu}$ of degree at most $D_{i}$. For $i \neq j$ we have $Z_{i} \cap Z_{j}=\emptyset$, hence the $f_{j \nu}$ have no common zero in $Z_{i}$. By Theorem 2.1 there exist polynomials $g_{\nu}$ with $\operatorname{deg}\left(g_{\nu} f_{j \nu}\right) \leq 2 D_{i} D_{j}^{m}$ such that

$$
\varphi_{i j}:=\sum_{\nu} g_{\nu} f_{j \nu}=1 \quad \text { on } \quad Z_{i} .
$$

It is easy to see that the desired idempotents can be defined as

$$
e_{i}:=\prod_{j<i} \varphi_{i j} \cdot \prod_{j>i} \varphi_{i j} .
$$


Their degrees satisfy

$$
\operatorname{deg} e_{i} \leq 2 D_{i} \sum_{j \neq i} D_{j}^{m} \leq 2 D_{i}\left(\sum_{j \neq i} D_{j}\right)^{m}=2 D_{i}\left(D-D_{i}\right)^{m} .
$$

A small curve discussion shows that the last expression, as a function of $D_{i}$, is maximal for $D_{i}=\frac{D}{m+1}$, which implies $\operatorname{deg} e_{i} \leq \frac{2}{m+1}\left(\frac{m}{m+1}\right)^{m} D^{m+1} \leq D^{m+1}$ for $m \geq 1$.

This result gives a very good bound for small dimensions, say for curves. However, Kollár's effective Nullstellensatz for arbitrary ideals implies a bound which is quadratic in the degree and therefore gives better results for larger dimensions.

Proposition 3.2. Let $X \subseteq \mathbb{A}^{n}$ be a closed variety of degree $D$. Then

$$
\operatorname{deg}\left(H_{\mathrm{dR}}^{0}(X)\right) \leq \frac{n+1}{4} D^{2} .
$$

Proof. By Theorem 2.2 there exist polynomials $\varphi_{i j} \in I\left(Z_{i}\right)$ and $\psi_{i j} \in I\left(Z_{j}\right)$ for $i \neq j$ such that

$$
\operatorname{deg}\left(\varphi_{i j}\right), \operatorname{deg}\left(\psi_{i j}\right) \leq(n+1) D_{i} D_{j} \quad \text { and } \quad \varphi_{i j}+\psi_{i j}=1 .
$$

Now the desired idempotents can be defined as

$$
e_{i}:=\prod_{j<i} \varphi_{j i} \cdot \prod_{j>i} \psi_{i j}
$$

Their degrees satisfy

$$
\operatorname{deg} e_{i} \leq(n+1) D_{i} \sum_{j \neq i} D_{j}=(n+1) D_{i}\left(D-D_{i}\right) \leq(n+1)(D / 2)^{2} .
$$

Remark 3.3. In [34] we have proved that for a hypersurface $X$ the factor $n+1$ in (8) can be dropped.

Example 3.4. This example shows that the bound (8) is sharp up to the factor $n+1$. It is derived from Example 2.3 of [25], which goes back to Masser, Philippon, and Brownawell [6].

Let $d \geq 1$. Consider the polynomials

$$
f_{1}:=X_{1}, \quad f_{2}:=X_{2} X_{3}^{d-1}-1, \quad f_{3}:=X_{1} X_{3}^{d-1}-X_{2}^{d},
$$

and set $Z_{1}:=\mathcal{Z}\left(f_{1}, f_{2}\right)$ and $Z_{2}:=\mathcal{Z}\left(f_{3}\right)$ in $\mathbb{A}^{3}$. Clearly, both $Z_{1}$ and $Z_{2}$ are smooth irreducible varieties of degree $d$ that do not intersect. Consequently, they are the connected components of $X:=Z_{1} \cup Z_{2}$, and $D:=\operatorname{deg} X=2 d$. Now consider the projective closure $\bar{X}=\bar{Z}_{1} \cup \bar{Z}_{2}$. Let $F_{i} \in k\left[X_{0}, \ldots, X_{3}\right]$ denote the homogenization of $f_{i}$, i.e,

$$
F_{1}=X_{1}, \quad F_{2}:=X_{2} X_{3}^{d-1}-X_{0}^{d}, \quad F_{3}=X_{1} X_{3}^{d-1}-X_{2}^{d} .
$$


One easily checks that $I\left(\bar{Z}_{1}\right)=\left(F_{1}, F_{2}\right)$ and $I\left(\bar{Z}_{2}\right)=\left(F_{3}\right)$. Now let $e_{1}, e_{2} \in$ $k\left[X_{1}, X_{2}, X_{3}\right]$ denote the idempotents of $Z_{1}$ and $Z_{2}, \delta:=\max \left\{\operatorname{deg} e_{1}, \operatorname{deg} e_{2}\right\}$, and $E_{1}, E_{2}$ their homogenizations w.r.t. degree $\delta$, i.e., $E_{i}=X_{0}^{\delta} e_{i}\left(X / X_{0}\right)$. Then we have

$$
E_{1}+E_{2}=X_{0}^{\delta} \text { on } \bar{X}, \quad E_{1}=0 \text { on } \bar{Z}_{2}, \quad E_{2}=0 \text { on } \bar{Z}_{1},
$$

hence $X_{0}^{\delta} \in I\left(\bar{Z}_{1}\right)+I\left(\bar{Z}_{2}\right)$. It follows that its image in

$$
k\left[X_{0}, \ldots, X_{3}\right] /\left(F_{1}, F_{2}, F_{3}, X_{3}-1\right) \simeq k\left[X_{0}\right] /\left(X_{0}^{d^{2}}\right)
$$

is also zero, so that

$$
\max \left\{\operatorname{deg} e_{1}, \operatorname{deg} e_{2}\right\}=\delta \geq d^{2}=D^{2} / 4 .
$$

\section{Some Reductions}

Our aim is to show that it is sufficient to prove the claimed bounds for certain irreducible hypersurfaces. In this section we first reduce to the irreducible case, and then effectively construct a cover of $X$ by affine open patches which are isomorphic to hypersurfaces. In the next section, we will show how degree bounds on those patches yield a bound for $H_{\mathrm{dR}}^{\bullet}(X)$.

The reduction to the irreducible case follows from the effective characterization of the connected components discussed in $\S 3$.

Corollary 4.1. If $X \subseteq \mathbb{A}^{n}$ is a smooth closed variety of degree $D$ with irreducible components $Z_{i}$ and $N:=\min \left\{(n+1) D^{2} / 4, D^{m+1}\right\}$, then

$$
\operatorname{deg}\left(H_{\mathrm{dR}}^{\bullet}(X)\right) \leq \max _{i}\left\{\operatorname{deg}\left(H_{\mathrm{dR}}^{\bullet}\left(Z_{i}\right)\right)\right\}+N
$$

Proof. Since the irreducible components of $X$ coincide with its connected components, the restrictions of differential forms to the $Z_{i}$ induce an isomorphism

$$
\left.H_{\mathrm{dR}}^{\bullet}(X) \simeq \bigoplus_{i} H_{\mathrm{dR}}^{\bullet}\left(Z_{i}\right)\right)
$$

Let $e_{i} \in k[X]$ denote the idempotent corresponding to $Z_{i}$. Since $e_{i}$ is 1 on $Z_{i}$ and 0 on $X \backslash Z_{i}$, the embedding $H_{\mathrm{dR}}^{\bullet}\left(Z_{i}\right) \hookrightarrow H_{\mathrm{dR}}^{\bullet}(X)$ is induced by the map $\omega \mapsto e_{i} \omega$, so that Propositions 3.1 and 3.2 imply the claim.

It is well known that each irreducible variety $X$ is birational to a hypersurface $Y$. By Zariski's Main Theorem, there exist open dense subsets $U \subseteq X$ and $V \subseteq Y$ which are isomorphic. Now we make this construction effective and obtain the result that degree and order bounds for $H_{\mathrm{dR}}^{\bullet}(V)$ imply such bounds for $H_{\mathrm{dR}}^{\bullet}(U)$.

The following lemma essentially consists of the construction of a geometric resolution $[14,31]$. 
Lemma 4.2. Let $X \subseteq \mathbb{A}^{n}$ be a closed irreducible subvariety of dimension $m<n$ and $\Xi \subseteq X$ be a finite subset. Then there exists a linear coordinate transformation after which $X$ is in Noether normal position with algebraically independent variables $X_{1}, \ldots, X_{m}$ and a polynomial $f \in k\left[X_{1}, \ldots, X_{m+1}\right]$ such that

(i) $f$ is irreducible and monic in $X_{m+1}$,

(ii) $\operatorname{deg} f \leq \operatorname{deg} X$,

(iii) $Y:=\mathcal{Z}(f) \subseteq \mathbb{A}^{m+1}$ is the closure of the image of $X$ under the projection $\pi: \mathbb{A}^{n} \rightarrow \mathbb{A}^{m+1},\left(x_{1}, \ldots, x_{n}\right) \mapsto\left(x_{1}, \ldots, x_{m+1}\right)$,

(iv) with $g:=\frac{\partial f}{\partial X_{m+1}}$ the projection $\pi$ restricts to an isomorphism

$$
U:=X \backslash \mathcal{Z}(g) \stackrel{\simeq}{\longrightarrow} V:=Y \backslash \mathcal{Z}(g), \quad \text { and }
$$

(v) $g(\xi) \neq 0$ for all $\xi \in \Xi$.

Proof. Let $A=R / I(X)$ be the coordinate ring of $X$. A linear coordinate transformation brings $X$ into Noether normal position, i.e., we assume that the ring extension $k\left[X_{1}, \ldots, X_{m}\right] \hookrightarrow A$ is integral. Let $r:=n-m$. For $\lambda=\left(\lambda_{1}, \ldots, \lambda_{r}\right) \in k^{r}$ let $u_{\lambda}:=\lambda_{1} X_{m+1}+\cdots+\lambda_{r} X_{n}$. Then there exists a monic polynomial $f_{\lambda} \in k\left[X_{1}, \ldots, X_{m}\right][T]$ with $f_{\lambda}\left(u_{\lambda}\right)=0$ in $A$. Since $A$ is a domain, we can assume $f_{\lambda}$ to be irreducible. Let $\pi_{\lambda}: X \rightarrow \mathbb{A}^{m+1}$ be the map $\left(x_{1}, \ldots, x_{n}\right) \mapsto\left(x_{1}, \ldots, x_{m}, u_{\lambda}\right)$. Obviously, $f_{\lambda}$ vanishes on the irreducible hypersurface $\pi_{\lambda}(X)$ of $\mathbb{A}^{m+1}$, so it is a reduced equation for it. It follows $\operatorname{deg} f_{\lambda}=\operatorname{deg} \pi_{\lambda}(X) \leq \operatorname{deg} X$ [23, Lemma 2]. Now we consider $\lambda_{1}, \ldots, \lambda_{r}$ as variables and argue as above over the field $k_{\lambda}:=\overline{k\left(\lambda_{1}, \ldots, \lambda_{r}\right)}$. It is shown in $[15,32]$ that $f_{\lambda} \in k\left[\lambda, X_{1}, \ldots, X_{m}, T\right]$. Now set $g_{\lambda}:=\frac{\partial f_{\lambda}}{\partial T}$. Differentiating the equation $f_{\lambda}\left(u_{\lambda}\right)=0$ with respect to $\lambda_{i}$, the chain rule yields

$$
\frac{\partial f_{\lambda}}{\partial \lambda_{i}}\left(u_{\lambda}\right)+\frac{\partial f_{\lambda}}{\partial T}\left(u_{\lambda}\right) \frac{\partial u_{\lambda}}{\partial \lambda_{i}}=\frac{\partial f_{\lambda}}{\partial \lambda_{i}}\left(u_{\lambda}\right)+g_{\lambda}\left(u_{\lambda}\right) X_{m+i}=0, \quad 1 \leq i \leq r .
$$

We choose $\lambda \in k^{r}$ such that $g_{\lambda}\left(\xi_{1}, \ldots, \xi_{m}, u_{\lambda}(\xi)\right) \neq 0$ for all $\xi=\left(\xi_{1}, \ldots, \xi_{n}\right) \in$ $\Xi$ and denote $f:=f_{\lambda}, g:=g_{\lambda}, w_{i}:=\frac{\partial f_{\lambda}}{\partial \lambda_{i}}$. By another linear coordinate transformation we can assume $u_{\lambda}=X_{m+1}$. It follows that the map

$$
\mathcal{Z}(f) \backslash \mathcal{Z}(g) \rightarrow X \backslash \mathcal{Z}(g), \quad x=\left(x_{1}, \ldots, x_{m+1}\right) \mapsto\left(x,-\frac{w_{2}(x)}{g(x)}, \ldots,-\frac{w_{r}(x)}{g(x)}\right)
$$

is an inverse of the projection, which concludes the proof.

Remark 4.3. If $X \subseteq \mathbb{A}^{n}$ is smooth, then $V$ from Lemma 4.2 is also smooth.

Corollary 4.4. In the situation of Lemma 4.2, consider the open subsets $U^{\prime}:=$ $U \backslash \mathcal{Z}(h) \subseteq X$, where $h \in R$, and $V^{\prime}:=\pi\left(U^{\prime}\right) \subseteq Y$. Then there exists $H \in k\left[X_{1}, \ldots, X_{m+1}\right]$ with $\operatorname{deg} H \leq \operatorname{deg} h \operatorname{deg} X$ such that $k\left[V^{\prime}\right]=k[Y]_{g H}$. Moreover, the isomorphism $\varphi: k[Y]_{g H}=k\left[V^{\prime}\right] \stackrel{\widetilde{\rightrightarrows}}{\rightarrow} k\left[U^{\prime}\right]=k[X]_{g h}$ satisfies

$$
\varphi\left(F^{s, d} k[Y]_{g H}\right) \subseteq F^{s(\operatorname{deg} h+1), d} k[X]_{g h} .
$$


Proof. By the proof of Lemma 4.2, there exist $w_{i} \in k\left[X_{1}, \ldots, X_{m+1}\right]$ of degree at most $\operatorname{deg} f$ such that

$$
X_{m+i}=-w_{i} / g \quad \text { in } \quad k[X]_{g} \quad \text { for } \quad 1 \leq i \leq r=n-m .
$$

Thus, the preimage of $h$ under the isomorphism $k[Y]_{g} \stackrel{\simeq}{\longrightarrow} k[X]_{g}$ is the residue class of the rational function

$$
\widetilde{h}:=h\left(X_{1}, \ldots, X_{m},-\frac{w_{1}}{g}, \ldots,-\frac{w_{r}}{g}\right)=H\left(X_{1}, \ldots, X_{m+1}\right) / g^{\operatorname{deg} h},
$$

where $H \in k\left[X_{1}, \ldots, X_{m+1}\right]$. It follows $k\left[V^{\prime}\right]=k[Y]_{g H}$. Since $\operatorname{deg}\left(\frac{w_{i}}{g}\right) \leq 1$, we have

$$
\operatorname{deg} H=\operatorname{deg} \widetilde{h}+\operatorname{deg} h \operatorname{deg} g \leq \operatorname{deg} h(1+\operatorname{deg} g)=\operatorname{deg} h \operatorname{deg} f \leq \operatorname{deg} h \operatorname{deg} X .
$$

An element of $F^{s, d} k[Y]_{g H}$ is the residue class of a rational function $\frac{a}{(g H)^{s}}$, where $a \in k\left[X_{1}, \ldots, X_{m+1}\right]$ with $\operatorname{deg} a \leq d+s(\operatorname{deg} g+\operatorname{deg} H)$. The isomorphism $\varphi$ identifies $H$ with $g^{\operatorname{deg} h} h$, so in $k[X]_{g h}$ we have

$$
\frac{a}{(g H)^{s}} \equiv \frac{a}{\left(g \cdot g^{\operatorname{deg} h} h\right)^{s}}=\frac{a h^{s \operatorname{deg} h}}{(g h)^{s(\operatorname{deg} h+1)}} .
$$

Since

$$
\begin{aligned}
\operatorname{deg}\left(\frac{a}{\left(g \cdot g^{\operatorname{deg} h} h\right)^{s}}\right) & =\operatorname{deg} a-s(\operatorname{deg} h+(\operatorname{deg} h+1) \operatorname{deg} g) \\
& \leq d+s(\operatorname{deg} g+\operatorname{deg} h \operatorname{deg} f-\operatorname{deg} h-\operatorname{deg} h \operatorname{deg} g-\operatorname{deg} g) \\
& \leq d+s \operatorname{deg} h \underbrace{\operatorname{deg} f-1-\operatorname{deg} g)}_{=0}=d,
\end{aligned}
$$

we have $\varphi\left(\overline{\frac{a}{(g H)^{s}}}\right) \in F^{s(\operatorname{deg} h+1), d} k[X]_{g h}$.

Corollary 4.5. Let $X \subseteq \mathbb{A}^{n}$ be a closed irreducible variety of dimension $m$. Then there exist principal open subsets $U_{0}, \ldots, U_{m}$ covering $X$, such that each $U_{i}$ is isomorphic to an open subset of a hypersurface described as in Lemma 4.2.

Proof. We apply Lemma 4.2 successively to construct the $U_{i}$. We start with an arbitrary one-point set $\Xi_{0}=\{\xi\} \subseteq X$ to obtain $U_{0}$. Having constructed $U_{0}, \ldots, U_{i-1}$, let $\Xi_{i}$ contain a point from each irreducible component of $X \backslash$ $\bigcup_{0 \leq j<i} U_{j}$, so that one obtains $U_{i}$ with

$$
\operatorname{dim}\left(X \backslash \bigcup_{j=0}^{i-1} U_{j}\right)>\operatorname{dim}\left(X \backslash \bigcup_{j=0}^{i} U_{j}\right)
$$

It follows that $\operatorname{dim} X \backslash \bigcup_{0 \leq j \leq m} U_{j}=-1$, hence $\bigcup_{i=0}^{m} U_{i}=X$. 


\section{Effective Čech and Hypercohomology}

In this section we show how the cohomologies of the open patches of Corollary 4.5 fit together to give effective descriptions of the cohomology of $X$. This is done via effective hypercohomology, for which we first need to make Čech cohomology effective.

So let $X \subseteq \mathbb{A}^{n}$ be a closed variety, and let $\mathcal{F}$ be a coherent sheaf on $X$. Since $X$ is affine, we have

$$
H^{0}(X, \mathcal{F})=\Gamma(X, \mathcal{F}), \quad H^{q}(X, \mathcal{F})=0 \quad \text { for } \quad q>0 .
$$

Now let $A:=k[X]$ be the coordinate ring of $X$, and let $M$ be a finitely generated $A$-module such that $\mathcal{F}=\widetilde{M}$. Furthermore, let $\mathcal{U}=\left\{U_{i} \mid 0 \leq i \leq t\right\}$ be a cover of $X$ by principal open subsets $U_{i}=X \backslash \mathcal{Z}\left(g_{i}\right)$, where $g_{i} \in R$ is a non-zerodivisor on $A$. By $\S 2.4$, the sheaf cohomology of $\mathcal{F}$ can be computed as the Čech cohomology with respect to $\mathcal{U}$. Using $\S 2.3$, the Cech complex $C^{\bullet}=C^{\bullet}(\mathcal{U}, \mathcal{F})$ can be explicitly descibed as follows. For a set of indices $0 \leq i_{0}, \ldots, i_{q} \leq t$ set $g_{i_{0} \cdots i_{q}}:=g_{i_{0}} \cdots g_{i_{q}}$. Then

$$
C^{q}=\bigoplus_{0 \leq i_{0}<\ldots<i_{q} \leq t} M_{g_{i_{0} \ldots i_{q}}}, \quad 0 \leq q \leq t .
$$

The properties (10) mean that the augmented complex

$$
0 \longrightarrow C^{-1}:=M \stackrel{\delta^{-1}}{\longrightarrow} C^{0} \stackrel{\delta^{0}}{\longrightarrow} C^{1} \stackrel{\delta^{1}}{\longrightarrow} \cdots \stackrel{\delta^{t-1}}{\longrightarrow} C^{t} \longrightarrow 0
$$

is exact, where $\delta^{-1}$ is induced by the restriction. Note that $\delta^{-1}$ yields the isomorphism $M \simeq H^{0}(X, \mathcal{F})$.

The point of this section is to study degree and order bounds for a preimage under $\delta^{\bullet}$. So we consider a filtration

$$
\cdots \subseteq F^{d} M \subseteq F^{d+1} M \subseteq \cdots \subseteq M
$$

on $M$ which is compatible with $D^{\bullet} A$. Recall from $\S 2.1$ that there is an induced double filtration $F^{\bullet \bullet \bullet} M_{g_{i_{0} \cdots i_{q}}}$. These double filtrations extend to the spaces $C^{q}$ by setting

$$
F^{s, d} C^{q}:=\bigoplus_{0 \leq i_{0}<\cdots<i_{q} \leq t} F^{s, d} M_{g_{i_{0}} \cdots g_{i_{q}}}
$$

Since the restriction does increase neither degree nor order, we have $\delta^{q}\left(F^{s, d} C^{q}\right) \subseteq$ $F^{s, d} C^{q+1}$.

Lemma 5.1. Let $\operatorname{deg} X=D$, $\operatorname{deg} g_{i} \leq d_{1}$, and $N:=2 D\left(s d_{1}\right)^{m}$. Then

$$
F^{s, d} C^{q} \cap \operatorname{ker} \delta^{q} \subseteq \delta^{q-1}\left(F^{s, d+N} C^{q-1}\right) \quad \text { for all } \quad 0 \leq q \leq t .
$$


Proof. For $\omega=\left(\omega_{i_{0} \cdots i_{q}}\right)_{i_{0}<\cdots<i_{q}} \in F^{s, d} C^{q} \cap \operatorname{ker} \delta^{q}$ we write $\omega_{i_{0} \cdots i_{q}}=\frac{\alpha_{i_{0} \cdots i_{q}}}{g_{i_{0} \cdots i_{q}}}$, where $\alpha_{i_{0} \cdots i_{q}} \in M$ with $\operatorname{deg} \alpha_{i_{0} \cdots i_{q}} \leq d+s \operatorname{deg} g_{i_{0} \cdots i_{q}}$. The assumption $\delta^{q}(\omega)=0$ implies

$$
\sum_{\nu}(-1)^{\nu} g_{i_{\nu}}^{s} \alpha_{i_{0} \cdots \hat{i_{\nu}} \cdots i_{q+1}}=0 \text { for all } 0 \leq i_{0}<\cdots<i_{q+1} \leq t .
$$

Since $\mathcal{U}$ is a cover of $X$, the $g_{0}^{s}, \ldots, g_{t}^{s}$ have no common zero in $X$. Thus, by Theorem 2.1 there are $h_{0}, \ldots, h_{t} \in A$ with $\sum_{i} h_{i} g_{i}^{s}=1$ and $\operatorname{deg}\left(h_{i} g_{i}^{s}\right) \leq N$. Now we let $\eta=\left(\eta_{i_{0} \cdots i_{q-1}}\right) \in C^{q-1}$ with

$$
\eta_{i_{0} \cdots i_{q-1}}:=\frac{1}{g_{i_{0} \cdots i_{q-1}}^{s}} \sum_{i=0}^{t} h_{i} \alpha_{i, i_{0} \cdots i_{q-1}} \quad \text { for all } \quad 0 \leq i_{0}<\cdots<i_{q-1} \leq t .
$$

Here we define $\alpha_{i, i_{0} \cdots i_{q-1}}$ to be zero, if $i \in\left\{i_{0} \cdots i_{q-1}\right\}$, and $\varepsilon \alpha_{j_{0} \cdots j_{q}}$ otherwise, where $\left\{i_{0}, \ldots, i_{q-1}, i\right\}=\left\{j_{0}<\cdots<j_{q}\right\}$ and $\varepsilon$ is the sign of the permutation sorting $\left(i_{0}, \ldots, i_{q-1}, i\right)$. Obviously, we have ord $\eta_{i_{0} \cdots i_{q-1}} \leq s$. To bound its degree, note that for all $i$

$\operatorname{deg}\left(h_{i} \alpha_{i, i_{0} \cdots i_{q-1}}\right) \leq N-s \operatorname{deg} g_{i}+d+s \operatorname{deg} g_{i, i_{0} \cdots i_{q-1}}=N+d+s \operatorname{deg} g_{i_{0} \cdots i_{q-1}}$, which implies $\operatorname{deg} \eta \leq N+d$.

Finally, we have

$$
\begin{aligned}
\delta^{q-1}(\eta)_{i_{0} \cdots i_{q}} & =\sum_{\nu=0}^{q}(-1)^{\nu} \eta_{i_{0} \cdots \widehat{i_{\nu}} \cdots i_{q}} \mid U_{i_{0} \cdots i_{q}} \\
& =\sum_{\nu=0}^{q}(-1)^{\nu} \frac{1}{g_{i_{0} \cdots \hat{i_{\nu}} \cdots i_{q}}^{s}} \sum_{i=0}^{t} h_{i} \alpha_{i, i_{0} \cdots \widehat{i_{\nu}} \cdots i_{q}} \\
& =\frac{1}{g_{i_{0} \cdots i_{q}}^{s}} \sum_{i=0}^{t} h_{i} \sum_{\nu=0}^{q}(-1)^{\nu} g_{i_{\nu}}^{s} \alpha_{i, i_{0} \cdots \widehat{i_{\nu}} \cdots i_{q}} \\
& \stackrel{(11)}{=} \frac{1}{g_{i_{0} \cdots i_{q}}^{s}} \sum_{i=0}^{t} h_{i} g_{i}^{s} \alpha_{i_{0} \cdots i_{q}} \\
& =\omega_{i_{0} \cdots i_{q}},
\end{aligned}
$$

hence $\delta^{q-1}(\eta)=\omega$.

Next we will bound the degree and order of the hypercohomology of a complex of coherent sheaves, given that we have degree and order bounds for the cohomologies of the open patches. This can be formulated in a general setting.

Lemma 5.2. Let $C^{\bullet \bullet}$ be a first quadrant double complex, where each $C^{p, q}$ is equipped with a double filtration $F^{\bullet \bullet \bullet} C^{p, q}$. Then we have

$$
\bigcap_{p+q=\ell} B\left(F^{\bullet \bullet \bullet} H^{p}\left(C^{\bullet, q}\right)\right) \subseteq B\left(H^{\ell}\left(\operatorname{tot}^{\bullet}\left(C^{\bullet, \bullet}\right)\right)\right) \quad \text { for all } \quad \ell \geq 0 .
$$


Proof. The second spectral sequence ${ }_{I I} E_{r}$ of the double complex $C^{\bullet}, \bullet$ has first term

$$
{ }_{I I} E_{1}^{p, q}=H^{p}\left(C^{\bullet, q}\right) .
$$

Note that the double filtration on $C^{\bullet} \bullet \bullet$ induces a double filtration on the spectral sequence. Each term of the spectral sequence is a cohomology of the previous one realized as a subquotient, thus

$$
B\left(F^{\bullet, \bullet}{ }_{I I} E_{r}^{p, q}\right) \subseteq B\left(F^{\bullet, \bullet}{ }_{I I} E_{r+1}^{p, q}\right) \quad \text { for all } \quad r \geq 1 .
$$

Moreover, since the double complex is bounded, the spectral sequence collapses at a finite level, i.e., there exists $r$ such that ${ }_{I I} E_{r}={ }_{I I} E_{\infty}$. By convergence we have

$$
H^{\ell}\left(\operatorname{tot}^{\bullet}\left(C^{\bullet, \bullet}\right)\right) \simeq \bigoplus_{p+q=\ell}{ }_{I I} E_{\infty}^{p, q} .
$$

Finally, since this isomorphism respects the double filtrations $F^{\bullet \bullet \bullet} C^{p, q}$, we conclude

$$
B\left(H^{\ell}\left(\operatorname{tot}^{\bullet}\left(C^{\bullet \bullet \bullet}\right)\right)\right)=\bigcap_{p+q=\ell} B\left(F^{\bullet, \bullet}{ }_{I I} E_{\infty}^{p, q}\right),
$$

which implies the claim.

Now consider a bounded complex of coherent sheaves

$$
\mathcal{F}^{\bullet}: 0 \longrightarrow \mathcal{F}^{0} \stackrel{\mathrm{d}}{\longrightarrow} \mathcal{F}^{1} \stackrel{\mathrm{d}}{\longrightarrow} \cdots \stackrel{\mathrm{d}}{\longrightarrow} \mathcal{F}^{u} \longrightarrow 0
$$

on $X$, and let $M^{p}$ be finitely generated $A$-modules with $\mathcal{F}^{p}=\widetilde{M}^{p}$. Furthermore, let $\mathcal{U}=\left\{U_{i} \mid 0 \leq i \leq t\right\}$ be a cover of $X$ by principal open subsets $U_{i}=$ $X \backslash \mathcal{Z}\left(g_{i}\right)$ as above. Recall from $\S 2.5$ that the hypercohomology $\mathbb{H}^{\ell}\left(X, \mathcal{F}^{\bullet}\right)$ can be computed in two ways, namely as the cohomology of the total complex tot $\left.C^{\bullet} \bullet \bullet(\mathcal{U}, \mathcal{F})\right)$, and as the cohomology of the complex of global sections $M^{\bullet}$. Of course, the latter is much simpler, which is usually taken as an argument that one does not need hypercohomology in the affine setting. However, we want to go through these arguments to bound degrees. Assume that we have filtrations

$$
\cdots \subseteq F^{d} M^{p} \subseteq F^{d+1} M^{p} \subseteq \cdots \subseteq M^{p},
$$

such that the differential d satisfies

$$
\mathrm{d}\left(F^{s, d} M_{g}^{p}\right) \subseteq F^{s+1, d} M_{g}^{p}
$$

with respect to the induced double filtration on the local sections. As above, the Čech double complex and its total complex inherit this double filtration.

Theorem 5.3. Let $\operatorname{deg} X=D$ and $\operatorname{deg} g_{i} \leq d_{1}$. Then for all $\ell$ and all

$$
(s, d) \in \bigcap_{p+q=\ell} \bigcap_{i_{0}<\cdots<i_{q}} B\left(H^{p}\left(M_{g_{i_{0} \cdots i_{q}}}^{\bullet}\right)\right)
$$

we have

$$
\operatorname{deg}\left(H^{\ell}\left(M^{\bullet}\right)\right) \leq d+2 D(\ell+1)(s+\ell)^{m} d_{1}^{m} .
$$


Proof. Fix $\ell$ and set $N:=2 D(s+\ell)^{m} d_{1}^{m}$. By Lemma 5.2 each cohomology class $c \in H^{\ell}\left(\operatorname{tot}^{\bullet}\left(C^{\bullet} \bullet \bullet\right)\right)$ can be represented by an element $\omega=\left(\omega_{0, \ell}, \omega_{1, \ell-1}, \ldots, \omega_{\ell, 0}\right)$, where

$$
\omega_{p, q} \in F^{s, d} C^{p, q} \text { for all } p+q=\ell .
$$

Since $d^{\text {tot }}(\omega)=0$, we have in particular $\delta^{\ell}\left(\omega_{0, \ell}\right)=0$, so $\omega_{0, \ell}$ defines an element in $H^{\ell}\left(X, \mathcal{F}^{0}\right)$. By Lemma 5.1 there exists

$$
\eta \in F^{s, d+N} C^{0, \ell-1}
$$

with $\delta^{\ell-1}(\eta)=\omega_{0, \ell}$. Consider

$$
\omega^{\prime}:=\left(0, \omega_{1, \ell-1}+(-1)^{\ell} \mathrm{d} \eta, \omega_{2, \ell-2}, \ldots, \omega_{\ell, 0}\right) .
$$

Since $\omega-\omega^{\prime}=\left(\omega_{0, \ell},(-1)^{\ell-1} \mathrm{~d} \eta, 0, \ldots, 0\right)=\mathrm{d}^{\text {tot }}(\eta, 0, \ldots, 0), \omega$ and $\omega^{\prime}$ define the same cohomology class $c$. Note that $\omega_{1, \ell-1}+(-1)^{\ell} \mathrm{d} \eta \in F^{s+1, d+N} C^{1, \ell-1}$. Continuing this way, after $\ell$ steps we have a representative of $c$ of the form $\omega^{\prime \prime}=\left(0, \ldots, 0, \omega_{\ell, 0}^{\prime \prime}\right)$, where

$$
\omega_{\ell, 0}^{\prime \prime} \in F^{s+\ell, d+\ell N} C^{\ell, 0}
$$

The closedness of $\omega^{\prime \prime}$ means that $\delta^{0}\left(\omega_{\ell, 0}^{\prime \prime}\right)=0$ and $\mathrm{d}\left(\omega_{\ell, 0}^{\prime \prime}\right)=0$. Applying Lemma 5.1 once more yields $\alpha \in F^{d+(\ell+1) N} M^{\ell}$ with $\delta^{-1}(\alpha)=\omega_{\ell, 0}^{\prime \prime}$. Moreover, $\mathrm{d}(\alpha)=0$, hence $\alpha$ defines a class in $H^{\ell}\left(M^{\bullet}\right)$, which is the image of $c$ under the isomorphism $H^{\ell}\left(\operatorname{tot}^{\bullet}\left(C^{\bullet} \bullet\right)\right) \simeq H^{\ell}\left(M^{\bullet}\right)$.

We close this section by discussing the case of an open subset of $X:=\mathbb{A}^{n}$. Let $Z=\mathcal{Z}\left(f_{0}, \ldots, f_{r}\right) \subseteq X$ be a closed subvariety and consider its complement $W:=X \backslash Z$. If $r>0$, then $W$ is not affine, and as a result its de Rham cohomology $H_{\mathrm{dR}}^{\bullet}(W)$ cannot be computed by global sections, so we have to use hypercohomology instead. The open subsets $U_{i}:=X \backslash \mathcal{Z}\left(f_{i}\right), 0 \leq i \leq r$, form an open cover of $W$ with corresponding Čech-de Rham double complex

$$
C^{p, q}=\bigoplus_{i_{0}<\cdots<i_{q}} \Omega_{W}^{p}\left(U_{i_{0} \cdots i_{q}}\right) \quad \text { for all } p, q \geq 0
$$

where $U_{i_{0} \cdots i_{q}}=U_{i_{0}} \cap \cdots \cap U_{i_{q}}$. The differentials d: $C^{p, q} \rightarrow C^{p+1, q}$ are induced by the exterior derivatives, and the differentials $\delta^{q}: C^{p, q} \rightarrow C^{p, q+1}$ are the Cech differentials. The de Rham cohomology of $W$ is the cohomology of the total complex $\operatorname{tot}^{\bullet}\left(C^{\bullet \bullet \bullet}\right)$, i.e.,

$$
H_{\mathrm{dR}}^{\ell}(W)=\mathbb{H}^{\ell}\left(W, \Omega_{W}^{\bullet}\right)=H^{\ell}\left(\operatorname{tot}^{\bullet}\left(C^{\bullet}, \bullet\right)\right) .
$$

Lemma 5.4. Equip each $C^{p, q}$ with the shifted double filtration $S^{s, d} C^{p, q}:=$ $F^{s-q, d-q} C^{p, q}$ for $s, d \in \mathbb{N}$. Then, with respect to the induced double filtration on the total complex, we have

$$
\ell \cdot(1,1) \in B\left(S^{\bullet \bullet \bullet} H_{\mathrm{dR}}^{\ell}(W)\right)
$$


Proof. By Lemma 2.4 we have with respect to the shifted double filtration

$\ell \cdot(1,1) \in B\left(S^{\bullet \bullet \bullet} H_{\mathrm{dR}}^{p}\left(U_{i_{0} \cdots i_{q}}\right)\right) \quad$ for all $\quad p+q=\ell, 0 \leq i_{0}<\cdots<i_{q} \leq r$.

Applying Lemma 5.2 implies the claim.

In the case of a complete intersection we are able to give a more succinct description of $H_{\mathrm{dR}}^{\bullet}(W)$ using sheaf cohomology as follows.

Lemma 5.5. Let $\operatorname{dim} Z=n-r-1$. Then, for $\ell>0$, the projection map

$$
\operatorname{tot}^{\ell}\left(C^{\bullet, \bullet}\right)=C^{\ell-r, r} \oplus \cdots \oplus C^{\ell, 0} \rightarrow C^{\ell-r, r}=\Omega_{A_{f_{0} \cdots f_{r}}^{\ell-r}}^{\ell}
$$

induces an isomorphism

$$
H_{\mathrm{dR}}^{\ell}(W) \stackrel{\simeq}{\longrightarrow} H^{\ell-r}\left(H^{r}\left(W, \Omega_{W}^{\bullet}\right)\right) .
$$

Proof. Let $\mathcal{F}$ be a locally free sheaf on $X$. The long exact sequence of local cohomology is

$$
\cdots \rightarrow H^{q-1}(X, \mathcal{F}) \rightarrow H^{q-1}(W, \mathcal{F} \mid W) \rightarrow H_{Z}^{q}(X, \mathcal{F}) \rightarrow H^{q}(X, \mathcal{F}) \rightarrow \cdots .
$$

Since $H^{q-1}(X, \mathcal{F})=0$ for $q>1$, we conclude

$$
H^{q-1}(W, \mathcal{F} \mid W) \simeq H_{Z}^{q}(X, \mathcal{F}) \text { for } \quad q>1 .
$$

Since $Z$ is a complete intersection of codimenion $r+1$, it follows from [19, Theorem 3.8], that $H_{Z}^{q}(X, \mathcal{F})=0$ for $q<r+1$. Furthermore, since $W=$ $X \backslash Z$ can be covered by $r+1$ affine open subsets, Čech cohomology implies $H_{Z}^{q}(X, \mathcal{F})=H^{q-1}(W, \mathcal{F} \mid W)=0$ for $q>r+1$. Thus

$$
H_{Z}^{q}(X, \mathcal{F})=0 \quad \text { for } \quad q \neq r+1 .
$$

The beginning of the long exact sequence together with (14) implies

$$
H^{0}(W, \mathcal{F} \mid W)=H^{0}(X, \mathcal{F}) .
$$

Now, the first spectral sequence of hypercohomology has first term

$$
{ }_{I} E_{1}^{p q}=H^{q}\left(W, \Omega_{W}^{p}\right) \quad \Rightarrow \quad H_{\mathrm{dR}}^{p+q}(W) .
$$

By (13), (14), and (15) we have

$$
{ }_{I} E_{1}^{p q}=\left\{\begin{aligned}
H^{0}\left(X, \Omega_{X}^{p}\right) & \text { if } q=0, \\
H^{r}\left(W, \Omega_{W}^{p}\right) & \text { if } q=r, \\
0 & \text { otherwise. }
\end{aligned}\right.
$$

Since $H_{\mathrm{dR}}^{p}(X)=H^{p}\left(H^{0}\left(X, \Omega_{X}^{p}\right)\right)=0$ for $p>0$, the second page ${ }_{I} E_{2}$ has differential zero, hence ${ }_{I} E_{2}={ }_{I} E_{\infty}$, and we conclude

$$
H_{\mathrm{dR}}^{\ell}(W)={ }_{I} E_{2}^{\ell-r, r}=H^{\ell-r}\left(H^{r}\left(W, \Omega_{W}^{\bullet}\right)\right) \quad \text { for } \quad \ell>0 .
$$

Lemmas 5.4 and 5.5 imply

Corollary 5.6.

$$
(\ell-r) \cdot(1,1) \in B\left(H^{\ell-r}\left(H^{r}\left(W, \Omega_{W}^{\bullet}\right)\right)\right)
$$




\section{Effective Gysin Sequence}

The main tool in our proof is the Gysin sequence which is the following

Theorem 6.1. Let $X$ be a smooth irreducible variety and $Z \subseteq X$ a smooth closed equidimensional subvariety of codimension $r$. Then there is an exact sequence

$$
\cdots \rightarrow H_{\mathrm{dR}}^{p}(X) \rightarrow H_{\mathrm{dR}}^{p}(X \backslash Z) \stackrel{\text { Res }}{\rightarrow} H_{\mathrm{dR}}^{p-2 r+1}(Z) \rightarrow H_{\mathrm{dR}}^{p+1}(X) \rightarrow \cdots
$$

Let us first record an easy consequence of the Gysin sequence for the case that $X$ is the affine space $\mathbb{A}^{n}$. Since $H_{\mathrm{dR}}^{p}(X)=0$ for $p>0$, Theorem 6.1 implies

Corollary 6.2. For a smooth closed equidimensional variety $Z \subseteq \mathbb{A}^{n}$ of codimension $r$ the residue map

$$
\operatorname{Res}: H_{\mathrm{dR}}^{p}\left(\mathbb{A}^{n} \backslash Z\right) \stackrel{\simeq}{\rightarrow} H_{\mathrm{dR}}^{p-2 r+1}(Z)
$$

is an isomorphism for all $p>0$.

An algebraic proof of Theorem 6.1 is given in [20]. Using this idea we will prove an effective version of it, i.e., we will describe the map Res explicitly on the level of differential forms, so that we can control its effect on their degrees.

However, our version only deals with the case of a smooth codimension 2 complete intersection of a very special type. In particular, we will study the following case. Denote $A:=k\left[X_{0}, \ldots, X_{n}\right]$, let $f, g \in R:=k\left[X_{1}, \ldots, X_{n}\right]$, and consider the regular sequence $f_{0}:=g X_{0}-1, f_{1}:=f$. Set $X:=\mathbb{A}^{n+1}$, $Z:=\mathcal{Z}\left(f_{0}, f_{1}\right)$ and denote its coordinate $\operatorname{ring}$ by $B:=k[Z]$. Assume that $f$ is irreducible and that $\frac{\partial f}{\partial X_{n}} \mid g$. Note that these assumptions imply that $Z$ is a smooth complete intersection in $X$ of codimension 2, and its vanishing ideal is $I:=I(Z)=\left(f_{0}, f_{1}\right)$, hence $B=A /\left(f_{0}, f_{1}\right)$.

Since in our case the complement $W:=X \backslash Z$ is not affine, its de Rham cohomology $H_{\mathrm{dR}}^{\bullet}(W)$ is described as in the last section. Recall that by Lemma 5.5 we have an isomorphism

$$
H_{\mathrm{dR}}^{p+1}(W) \stackrel{\simeq}{\longrightarrow} H^{p}\left(H^{1}\left(W, \Omega_{W}^{\bullet}\right)\right)=H^{p}\left(\frac{\Omega_{A_{f_{0} f_{1}}}^{\bullet}}{\Omega_{A_{f_{0}}}+\Omega_{A_{f_{1}}}^{\bullet}}\right) \quad \text { for } \quad p \geq 0 .
$$

The main result of this section is

Theorem 6.3. Let $f_{0}, f_{1} \in A$ be as above, and denote $d_{0}:=\operatorname{deg} f_{0}, d_{1}:=\operatorname{deg} f_{1}$. Then, under the identification (16), the map

$$
\text { Res: } H_{\mathrm{dR}}^{p+1}(W) \rightarrow H_{\mathrm{dR}}^{p-2}(Z) \quad \text { for } \quad p \geq 0
$$

is induced by a map $\Omega_{A_{f_{0} f_{1}}}^{p} \rightarrow \Omega_{B}^{p-2}$ which takes a p-form $\omega=\frac{\alpha}{\left(f_{0} f_{1}\right)^{s}}$ to a $(p-2)$-form $\operatorname{Res}(\omega)$ with

$$
\operatorname{deg} \operatorname{Res}(\omega) \leq\left(2 d_{0}-d_{1}+1\right)^{2 s-1} \operatorname{deg} \alpha .
$$


We fix the notations and assumptions of this theorem for the rest of the section. For its proof we will need the completion $\widehat{A}=\widehat{A}_{I}$ of $A$ with respect to $I$. Recall from $\S 2.2$ that $\widehat{A} \simeq A\left[\left[T_{0}, T_{1}\right]\right] /\left(T_{0}-f_{0}, T_{1}-f_{1}\right)$, so its elements are power series in $f_{0}, f_{1}$. Note, however, that these power series are not unique. E.g., $f_{0} \in A$ can be represented by the constant power series $f_{0}$ or by $T_{0}$. The crucial result for us is a lemma of Grothendieck stating that there is an algebra isomorphism $B\left[\left[T_{0}, T_{1}\right]\right] \rightarrow \widehat{A}$ (cf. [21, Lemma II,1.2]), which establishes a unique power series representation for the completion. We need to construct this isomorphism explicitly in order to bound degrees. The technical construction is in the following statement, which is a consequence of the fact that $B$ is a formally smooth $k$-algebra [17, Definition 19.3.1].

For a tuple $x=\left(x_{1}, \ldots, x_{n}\right)$ over an affine algebra $C$ we write $\operatorname{deg}(x):=$ $\max _{j} \operatorname{deg}\left(x_{j}\right)$ and use an analogous notation for the order. If $\psi: C \rightarrow D$ is a homomorphism, we write $\psi(x):=\left(\psi\left(x_{1}\right), \ldots, \psi\left(x_{n}\right)\right)$. For $x \in C$ we denote by $\bar{x}$ its image in any factor algebra of $C$.

Lemma 6.4. Let $N \in \mathbb{N}$ and $\psi: B \rightarrow A / I^{N}$ be an algebra homomorphism that lifts the identity $B \rightarrow B$, i.e., the composition $B \rightarrow A / I^{N} \rightarrow B$ is the identity. Then $\psi$ can be lifted to an algebra homomorphism $\widetilde{\psi}: B \rightarrow A / I^{N+1}$, i.e., the diagram

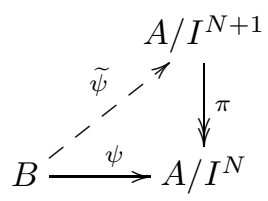

commutes.

Proof. Since the $k$-algebra $B$ is generated by $\bar{X}_{0}, \ldots, \bar{X}_{n}$, it is sufficient to define $\widetilde{\psi}$ on these elements. Choose $Y_{0}, \ldots, Y_{n} \in A$ such that $\psi\left(\bar{X}_{i}\right)=\bar{Y}_{i}$ in $A / I^{N}$ for all $0 \leq i \leq n$. Our aim is to define

$$
\widetilde{\psi}\left(\bar{X}_{i}\right):=\overline{Y_{i}+\sum_{\mu+\nu=N} a_{\mu \nu}^{(i)} f_{0}^{\mu} f_{1}^{\nu}} \text { for } \quad 0 \leq i \leq n
$$

with suitably chosen $a_{\mu \nu}^{(i)} \in A$. Then it is clear that $\pi \circ \widetilde{\psi}=\psi$. It remains to show that one can define $\widetilde{\psi}$ unambigously by (18). This means that we have to find $a_{\mu \nu}^{(i)}$ such that $f_{0}, f_{1}$ are mapped to zero in $A / I^{N+1}$. Set $Y:=\left(Y_{0}, \ldots, Y_{n}\right)$, $a_{\mu \nu}:=\left(a_{\mu \nu}^{(0)}, \ldots, a_{\mu \nu}^{(n)}\right)$, and look at the first condition

$$
f_{0} \mapsto \overline{f_{0}\left(Y+\sum_{\mu+\nu=N} a_{\mu \nu} f_{0}^{\mu} f_{1}^{\nu}\right)}=0 \quad \text { in } A / I^{N+1} .
$$

By the Taylor formula we have

$$
f_{0}\left(Y+\sum_{\mu+\nu=N} a_{\mu \nu} f_{0}^{\mu} f_{1}^{\nu}\right) \equiv f_{0}(Y)+\sum_{i=0}^{n} \frac{\partial f}{\partial X_{i}}(Y) \sum_{\mu+\nu=N} a_{\mu \nu}^{(i)} f_{0}^{\mu} f_{1}^{\nu} \quad\left(\bmod I^{N+1}\right) .
$$


Since $\overline{f_{0}(Y)}=\psi\left(\overline{f_{0}}\right)=0$ in $A / I^{N}$ and $I^{N}=\left(f_{0}^{N}, f_{0}^{N-1} f_{1}, \ldots, f_{0} f_{1}^{N-1}, f_{1}^{N}\right)$, there exist $p_{\mu \nu} \in A, \mu+\nu=N$, such that $f_{0}(Y)=\sum_{\mu+\nu=N} p_{\mu \nu} f_{0}^{\mu} f_{1}^{\nu}$ in $A$. Furthermore, since $\bar{Y}_{i}=\bar{X}_{i}$ in $B$, condition (19) is satisfied if

$$
p_{\mu \nu}+\sum_{i=0}^{n} \frac{\partial f_{0}}{\partial X_{i}} a_{\mu \nu}^{(i)} \equiv 0 \quad(\bmod I)
$$

Similarly, there exist $q_{\mu \nu} \in A, \mu+\nu=N$, such that $f_{1}(Y)=\sum_{\mu+\nu=N} q_{\mu \nu} f_{0}^{\mu} f_{1}^{\nu}$ in $A$, and $f_{1}$ is mapped to zero if

$$
q_{\mu \nu}+\sum_{i=0}^{n} \frac{\partial f_{1}}{\partial X_{i}} a_{\mu \nu}^{(i)} \equiv 0 \quad(\bmod I)
$$

In order to get very efficient degree bounds, we use the special form of the defining equations $f_{0}, f_{1}$. In particular, recall that there exists $h \in A$ such that $\frac{\partial f_{0}}{\partial X_{0}}=g=h \frac{\partial f_{1}}{\partial X_{n}}$. Also, note that $\frac{\partial f_{1}}{\partial X_{0}}=0$. This allows us to solve the linear system of equations over $B$ consisting of (20) and (21) as follows:

$$
\begin{aligned}
a_{\mu \nu}^{(n)} & :=-q_{\mu \nu} h X_{0}, \\
a_{\mu \nu}^{(i)} & :=0 \text { for } \quad 1 \leq i<n, \\
a_{\mu \nu}^{(0)} & :=-X_{0}\left(p_{\mu \nu}+\frac{\partial f_{0}}{\partial X_{n}} a_{\mu \nu}^{(n)}\right) .
\end{aligned}
$$

We check that these settings actually solve the system:

$$
q_{\mu \nu}+\sum_{i=0}^{n} \frac{\partial f_{1}}{\partial X_{i}} a_{\mu \nu}^{(i)} \equiv q_{\mu \nu}-\frac{\partial f_{1}}{\partial X_{n}} q_{\mu \nu} h X_{0} \equiv-q_{\mu \nu} f_{0} \equiv 0 \quad(\bmod I) .
$$

Moreover, we have

$$
\begin{aligned}
p_{\mu \nu}+\sum_{i=0}^{n} \frac{\partial f_{0}}{\partial X_{i}} a_{\mu \nu}^{(i)} & \equiv p_{\mu \nu}-\frac{\partial f_{0}}{\partial X_{0}} X_{0}\left(p_{\mu \nu}+\frac{\partial f_{0}}{\partial X_{n}} a_{\mu \nu}^{(n)}\right)+\frac{\partial f_{0}}{\partial X_{n}} a_{\mu \nu}^{(n)} \\
& \equiv-\left(p_{\mu \nu}+\frac{\partial f_{0}}{\partial X_{n}} a_{\mu \nu}^{(n)}\right) f_{0} \equiv 0 \quad(\bmod I)
\end{aligned}
$$

which concludes the proof.

Corollary 6.5. There exists an embedding $\psi: B \hookrightarrow \widehat{A}$ such that $\psi\left(\bar{X}_{i}\right)=$ $\sum_{\mu, \nu=0}^{\infty} a_{\mu \nu}^{(i)} f_{0}^{\mu} f_{1}^{\nu}$, where $a_{\mu \nu}^{(i)} \in A$ with

$$
\operatorname{deg} a_{\mu \nu}^{(i)} \leq 2 d_{0}-d_{1}+1 \quad \text { for all } \mu, \nu \in \mathbb{N}, 0 \leq i \leq n .
$$

Proof. We start with $\psi_{1}:=\mathrm{id}_{B}$ and apply Lemma 6.4 successively to construct the homomorphisms $\psi_{N}: B \rightarrow A / I^{N}, N \in \mathbb{N}$. Together they define a homomorphism $\psi: B \rightarrow \widehat{A}$, which is clearly injective. To prove the degree bound, 
denote by $Y^{(N)}=\left(Y_{0}^{(N)}, \ldots, Y_{n}^{(N)}\right) \in A^{n+1}$ representatives of $\psi_{N}\left(\bar{X}_{i}\right)=\overline{Y_{i}^{(N)}}$ in $A / I^{N}$. Furthermore, let $p_{\mu \nu}^{(N)}, q_{\mu \nu}^{(N)} \in A$ with

$$
f_{0}\left(Y^{(N)}\right)=\sum_{\mu+\nu=N} p_{\mu \nu}^{(N)} f_{0}^{\mu} f_{1}^{\nu}, \quad f_{1}\left(Y^{(N)}\right)=\sum_{\mu+\nu=N} q_{\mu \nu}^{(N)} f_{0}^{\mu} f_{1}^{\nu} .
$$

Then, equation (23) shows that

$$
\operatorname{deg} q_{\mu \nu}^{(N+1)} \leq \operatorname{deg} q_{\mu-1, \nu}^{(N)} \quad \text { for all } \quad N \geq 1 .
$$

Since $q_{01}^{(1)}=1$ and $q_{10}^{(1)}=0$, we conclude inductively that

$$
\operatorname{deg} q_{\mu \nu}^{(N)} \leq 0 \quad \text { for all } \quad N \geq 1 .
$$

Moreover, by (24) we have

$$
\operatorname{deg} p_{\mu \nu}^{(N+1)} \leq \max \left\{\operatorname{deg} p_{\mu-1, \nu}^{(N)}, d_{0}-1+\operatorname{deg} h+1\right\} .
$$

Using $p_{01}^{(1)}=0$ and $p_{10}^{(1)}=1$, an induction proves

$$
\operatorname{deg} p_{\mu \nu}^{(N)} \leq d_{0}+\operatorname{deg} h=2 d_{0}-d_{1} \quad \text { for all } \quad N \geq 1 .
$$

The solution (22) implies $\operatorname{deg} a_{\mu \nu}^{(n)}=d_{0}-d_{1}+1$, and hence for all $i$

$$
\operatorname{deg} a_{\mu \nu}^{(i)} \leq 1+\max \left\{2 d_{0}-d_{1}, d_{0}-1+d_{0}-d_{1}+1\right\}=2 d_{0}-d_{1}+1 .
$$

For $a \in \widehat{A}$ we write $\operatorname{deg}_{\mu \nu} a \leq \delta_{\mu \nu}$, if there exists a representation $a=$ $\sum_{\mu, \nu>0} a_{\mu \nu} f_{0}^{\mu} f_{1}^{\nu}$ with $\operatorname{deg} a_{\mu \nu} \leq \delta_{\mu \nu}$ for all $\mu, \nu \in \mathbb{N}$. Denote $\xi_{i}:=\psi\left(\bar{X}_{i}\right)$ and $\xi:=\left(\xi_{0}, \ldots, \xi_{n}\right)$. Then the degree bound of the previous corollary reads in this notation $\operatorname{deg}_{\mu \nu}(\xi) \leq 2 d_{0}-d_{1}+1=: \gamma$.

Remark 6.6. A straight-forward induction with respect to the degree of $p$ shows

$$
\operatorname{deg}_{\mu \nu} p(\xi) \leq \gamma \operatorname{deg} p \text { for all } p \in A .
$$

Corollary 6.7. For all $a=\sum_{\mu, \nu \geq 0} a_{\mu \nu} f_{0}^{\mu} f_{1}^{\nu} \in \widehat{A}$ there exist unique $b \in B$ and $c \in \widehat{I}=I \widehat{A}$ with $a=\psi(b)+c$. Furthermore, we have

$$
\operatorname{deg} b \leq \operatorname{deg} a_{00},
$$

and there exist $d, e \in \widehat{A}$ with $c=d f_{0}+e f_{1}$ and

$$
\begin{aligned}
\operatorname{deg}_{\mu \nu} d & \leq \max \left\{\operatorname{deg} a_{\mu+1, \nu}, \gamma \operatorname{deg} a_{00}\right\}, \\
\operatorname{deg}_{\mu \nu} e & \leq \max \left\{\operatorname{deg} a_{\mu, \nu+1}, \gamma \operatorname{deg} a_{00}\right\} .
\end{aligned}
$$


Proof. We have the exact sequence of $\widehat{A}$-modules

$$
0 \longrightarrow \widehat{I} \longrightarrow \widehat{A} \stackrel{\pi}{\longrightarrow} B \longrightarrow 0,
$$

which splits by the homomorphism $\psi$. For this reason, $\widehat{A} \simeq B \oplus \widehat{I}$, and the existence and uniqueness of the claimed representation follows. Note that if $a=\psi(b)+c$, then $b=\pi(a)=\pi\left(a_{00}\right)$. This implies (26).

Since $\psi$ and $\pi$ are $k$-algebra homomorphisms and $a_{00}$ is a polynomial, we have $\psi(b)=\psi\left(\pi\left(a_{00}\right)\right)=a_{00}(\xi)$, thus $c=a-a_{00}(\xi)$, and using (25) we conclude $\operatorname{deg}_{\mu, \nu} c \leq \max \left\{\operatorname{deg} a_{\mu \nu}, \gamma \operatorname{deg} a_{00}\right\}$, which yields (27).

Now we define the homomorphism

$$
\widehat{\psi}: B\left[\left[T_{0}, T_{1}\right]\right] \rightarrow \widehat{A}, \quad \sum_{\mu, \nu \geq 0} b_{\mu \nu} T_{0}^{\mu} T_{1}^{\nu} \mapsto \sum_{\mu, \nu \geq 0} \psi\left(b_{\mu \nu}\right) f_{0}^{\mu} f_{1}^{\nu} .
$$

Lemma 6.8. The homomorphism $\widehat{\psi}$ is an isomorphism. For $a \in A$ we have

$$
\operatorname{deg}_{\mu \nu} \widehat{\psi}^{-1}(a) \leq \gamma^{\mu+\nu} \operatorname{deg} a \text { for } \quad \mu, \nu \geq 0 .
$$

Proof. We first prove that $\widehat{\psi}$ is injective. It is clear that $\psi_{N}: B \rightarrow A / I^{N}=$ $\widehat{A} / \widehat{I}^{N}$ is injective for all $N \geq 1$. Note also, that by construction the diagram

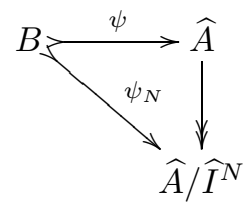

commutes. We show that for all $N \geq 1$ and all $b_{\mu \nu} \in B, \mu+\nu=N-1$, we have

$$
\sum_{\mu+\nu=N-1} \psi\left(b_{\mu \nu}\right) f_{0}^{\mu} f_{1}^{\nu} \equiv 0 \quad\left(\bmod \widehat{I}^{N}\right) \quad \Rightarrow \quad b_{\mu \nu}=0 \text { for all } \mu, \nu .
$$

For $N=1$ we have $0 \equiv \psi\left(b_{00}\right) \equiv \psi_{1}\left(b_{00}\right)(\bmod \widehat{I})$, hence $b_{00}=0$ by the injectivity of $\psi_{1}$. Now assume that (28) is true for some $N \geq 1$, and assume that $\sum_{\mu+\nu=N} \psi\left(b_{\mu \nu}\right) f_{0}^{\mu} f_{1}^{\nu} \equiv 0\left(\bmod \widehat{I}^{N+1}\right)$. Reducing $\bmod \left(f_{0}\right)$ yields $\psi\left(b_{0, N}\right) f_{1}^{N} \equiv$ $0\left(\bmod \left(f_{0}\right)\right)$. Since $f_{1}$ is a non-zerodivisor $\bmod \left(f_{0}\right)$, we conclude $\psi\left(b_{0, N}\right) \equiv 0$ $\left(\bmod \left(f_{0}\right)\right)$, thus $0 \equiv \psi\left(b_{0, N}\right) \equiv \psi_{1}\left(b_{0, N}\right)(\bmod \widehat{I})$. Injectivity of $\psi_{1}$ implies $b_{0, N}=0$. Now we write

$$
\psi\left(b_{N, 0}\right) f_{0}^{N}+\psi\left(b_{\mathbb{N}-1,1}\right) f_{0}^{N-1} f_{1}+\cdots+\psi\left(b_{1, N-1}\right) f_{0} f_{1}^{N-1}=f_{0} \cdot a,
$$

where $a \in \widehat{A}$ has the form of the assumption in (28). Since $f_{0} a \equiv 0\left(\bmod \widehat{I}^{N+1}\right)$, there exist $g_{\lambda \eta} \in \widehat{A}$ with $f_{0} a=\sum_{\lambda+\eta=N+1} g_{\lambda \eta} f_{0}^{\lambda} f_{1}^{\eta}$. Since $f_{1}$ is a nonzerodivisor $\bmod \left(f_{0}\right)$, we have $g_{0, N+1} \equiv 0\left(\bmod \left(f_{0}\right)\right)$, and since $f_{0}$ is a nonzerodivisor, we infer $a \equiv 0\left(\bmod \widehat{I}^{N}\right)$. The induction hypothesis implies $b_{\mu \nu}=0$ for all $\mu, \nu$ with $\mu+\nu=N$ and $\mu>0$, which completes the proof of (28). 
Now let $b=\sum_{\mu, \nu>0} b_{\mu \nu} T_{0}^{\mu} T_{1}^{\nu} \in B\left[\left[T_{0}, T_{1}\right]\right]$ with $\widehat{\psi}(b)=0$. We apply (28) inductively to conclude that $b=0$.

To show surjectivity, let $a \in \widehat{A}$ and construct a preimage $\sum_{\mu, \nu} b_{\mu \nu} T_{0}^{\mu} T_{1}^{\nu} \in$ $B\left[\left[T_{0}, T_{1}\right]\right]$ of $a$ under $\widehat{\psi}$. We find the $b_{\mu \nu}$ successively by applying Corollary 6.7. Let $b_{00} \in B$ and $d_{10}, d_{01} \in \widehat{A}$ with $a=\psi\left(b_{00}\right)+d_{10} f_{0}+d_{01} f_{1}$. Assume inductively, that for some $N \geq 1$ we have constructed $b_{\mu \nu} \in B$ for $\mu+\nu<N$, and $d_{\mu \nu} \in \widehat{A}$ for $\mu+\nu=N$, such that

$$
a=\sum_{\mu+\nu<N} \psi\left(b_{\mu \nu}\right) f_{0}^{\mu} f_{1}^{\nu}+\sum_{\mu+\nu=N} d_{\mu \nu} f_{0}^{\mu} f_{1}^{\nu} .
$$

Then, for all $\mu, \nu$ with $\mu+\nu=N$ we obtain from Corollary 6.7 elements $b_{\mu \nu} \in B$ and $d_{\mu \nu}^{0}, d_{\mu \nu}^{1} \in \widehat{A}$ such that

$$
d_{\mu \nu}=\psi\left(b_{\mu \nu}\right)+d_{\mu \nu}^{0} f_{0}+d_{\mu \nu}^{1} f_{1} .
$$

Plugging into (29) yields

$$
a=\sum_{\mu+\nu \leq N} \psi\left(b_{\mu \nu}\right) f_{0}^{\mu} f_{1}^{\nu}+\sum_{\mu+\nu=N}\left(d_{\mu \nu}^{0} f_{0}+d_{\mu \nu}^{1} f_{1}\right) f_{0}^{\mu} f_{1}^{\nu},
$$

which is of the form (29) for $N+1$ and hence completes the induction. We have $\sum_{\mu, \nu \geq 0} \psi\left(b_{\mu \nu}\right) f_{0}^{\mu} f_{1}^{\nu}=a$, since this equality holds modulo $\widehat{I}^{N}$ for all $N \geq 1$.

Now assume that $a \in A$. Then we claim

$$
\operatorname{deg}_{\lambda \eta}\left(d_{\mu \nu}\right) \leq \gamma^{\mu+\nu} \operatorname{deg} a \text { for all } \mu+\nu=N,
$$

which for $N=1$ follows directly from (27). Assuming (30) for some $N \geq 1$, (27) implies

$$
\operatorname{deg}_{\lambda \eta} d_{\mu \nu}^{0} \leq \max \left\{\operatorname{deg}_{\lambda+1, \eta} d_{\mu \nu}, \gamma \operatorname{deg}_{00} d_{\mu \nu}\right\} \leq \gamma^{\mu+\nu+1} \operatorname{deg} a,
$$

and $d_{\mu \nu}^{0}$ contributes to $d_{\mu+1, \nu}$. A similar estimate holds for $d_{\mu \nu}^{1}$, which completes the proof of $(30)$.

Finally, (30) and (26) yield

$$
\operatorname{deg}\left(b_{\mu \nu}\right) \leq \operatorname{deg}_{00} d_{\mu \nu} \leq \gamma^{\mu+\nu} \operatorname{deg} a \text { for all } \mu, \nu \in \mathbb{N} .
$$

Proof of Theorem 6.3. We prove the Theorem from scratch by constructing the residue map and checking that it is an isomorphism.

W.l.o.g. we can assume $p \geq 2$. As stated in the Theorem, we identify

$$
H_{\mathrm{dR}}^{p+1}(W) \simeq H^{p}\left(\frac{\Omega_{A_{f_{0} f_{1}}}^{\bullet}}{\Omega_{A_{f_{0}}}+\Omega_{A_{f_{1}}}^{\bullet}}\right) \text {. }
$$

Consider the map

$$
\lambda: H_{\mathrm{dR}}^{p-2}(Z) \rightarrow H^{p}\left(\frac{\Omega_{A_{f_{0} f_{1}}}}{\Omega_{A_{f_{0}}}+\Omega_{A_{f_{1}}}^{\bullet}}\right), \quad[\bar{\omega}] \mapsto\left[\overline{\frac{\mathrm{d} f_{0}}{f_{0}} \wedge \frac{\mathrm{d} f_{1}}{f_{1}} \wedge \omega}\right],
$$


where $\omega \in \Omega_{A}^{p-2}, \bar{\omega}$ denotes its image in $\Omega_{B}^{p-2}$, and $[\bar{\omega}]$ the cohomology class in $H_{\mathrm{dR}}^{p-2}(Z)$ it represents. A similar notation is used on the right hand side.

We first show that the map $\lambda$ is well-defined. If $\omega \in \Omega_{A}^{p-2}$ represents the zero cohomology class in $H_{\mathrm{dR}}^{p-2}(Z)$, then $\bar{\omega}=\mathrm{d} \bar{\eta}$ for some $\eta \in \Omega_{A}^{p-3}$. This means that $\omega-\mathrm{d} \eta$ is contained in the differential graded ideal generated by $I$ and $\mathrm{d} I$. Using the formula $\mathrm{d} f_{i} \wedge \alpha=\mathrm{d}\left(f_{i} \alpha\right)-f_{i} \mathrm{~d} \alpha$, we can assume that there exist $\alpha, \beta \in \Omega_{A}^{p-2}$ with $\omega-\mathrm{d} \eta=f_{0} \alpha+f_{1} \beta$. Then

$$
\begin{aligned}
\frac{\mathrm{d} f_{0}}{f_{0}} \wedge \frac{\mathrm{d} f_{1}}{f_{1}} \wedge \omega & =\mathrm{d} f_{0} \wedge \frac{\mathrm{d} f_{1}}{f_{1}} \wedge \alpha+\frac{\mathrm{d} f_{0}}{f_{0}} \wedge \mathrm{d} f_{1} \wedge \beta+\frac{\mathrm{d} f_{0}}{f_{0}} \wedge \frac{\mathrm{d} f_{1}}{f_{1}} \wedge \mathrm{d} \eta \\
& \equiv \mathrm{d}\left(\frac{\mathrm{d} f_{0}}{f_{0}} \wedge \frac{\mathrm{d} f_{1}}{f_{1}} \wedge \eta\right) \bmod \left(\Omega_{A_{f_{0}}}^{\bullet}+\Omega_{A_{f_{1}}}^{\bullet}\right)
\end{aligned}
$$

so it maps to zero in the cohomology on the right hand side. Furthermore, since $\mathrm{d} f_{i} / f_{i}$ is exact, one easily checks that $\lambda$ sends closed (exact) forms to closed (exact) ones.

The residue map will be the inverse of $\lambda$. To construct it, note that the isomorphism of Lemma 6.8 induces a homomorphism $\Omega_{A_{f_{0} f_{1}}^{\bullet}} \hookrightarrow \widehat{\Omega}_{\widehat{A}_{f_{0} f_{1}}} \stackrel{\simeq}{\longrightarrow}$ $\Omega_{B\left[\left[T_{0}, T_{1}\right]\right]_{T_{0} T_{1}}^{\bullet}}$ and as a result an isomorphism

$$
\vartheta: \frac{\Omega_{A_{f_{0} f_{1}}}^{\bullet}}{\Omega_{A_{f_{0}}}+\Omega_{A_{f_{1}}}^{\bullet}} \simeq \frac{\widehat{\Omega}_{\widehat{A}_{f_{0} f_{1}}}^{\bullet}}{\widehat{\Omega}_{\widehat{A}_{f_{0}}}^{\bullet}+\widehat{\Omega}_{\widehat{A}_{f_{1}}}^{\bullet}} \stackrel{\widehat{\Omega}_{B\left[\left[T_{0}, T_{1}\right]\right]_{T_{0} T_{1}}}^{\bullet}}{\longrightarrow} \frac{\widehat{\Omega}_{B\left[\left[T_{0}, T_{1}\right]\right]_{T_{0}}}^{\bullet}+\widehat{\Omega}_{B\left[\left[T_{0}, T_{1}\right]\right]_{T_{1}}}^{\bullet}}{\bullet} .
$$

Define

$$
\operatorname{Res}: H^{p}\left(\frac{\Omega_{A_{f_{0} f_{1}}}^{\bullet}}{\Omega_{A_{f_{0}}}+\Omega_{A_{f_{1}}}^{\bullet}}\right) \rightarrow H_{\mathrm{dR}}^{p-2}(Z)
$$

as follows. For a form $\omega \in \Omega_{A_{f_{0} f_{1}}}^{p}$ write

$$
\vartheta(\bar{\omega})=\sum_{\mu, \nu \geq 1}\left(\alpha_{\mu \nu}+\beta_{\mu \nu} \wedge \mathrm{d} T_{0}+\gamma_{\mu \nu} \wedge \mathrm{d} T_{1}+\delta_{\mu \nu} \wedge \mathrm{d} T_{0} \wedge \mathrm{d} T_{1}\right) T_{0}^{-\mu} T_{1}^{-\nu}
$$

with $\alpha_{\mu \nu}, \beta_{\mu \nu}, \gamma_{\mu \nu}, \delta_{\mu \nu} \in \Omega_{B}^{\bullet}$, only finitely many non-zero, and map $\operatorname{Res}([\bar{\omega}]):=$ $\left[\delta_{1,1}\right]$. For the proof that this maps closed forms to closed forms, see below.

To prove that this defines the inverse of $\lambda$, it suffices to show Res $\circ \lambda=$ id and $\lambda \circ$ Res $=$ id (in particular, this implies well-definedness). First, for $[\bar{\omega}] \in$ $H_{\mathrm{dR}}^{p-2}(Z)$ we have

$$
\operatorname{Res} \circ \lambda([\bar{\omega}])=\operatorname{Res}\left(\left[\overline{\frac{\mathrm{d} f_{0}}{f_{0}} \wedge \frac{\mathrm{d} f_{1}}{f_{1}} \wedge \omega}\right]\right)=[\bar{\omega}],
$$

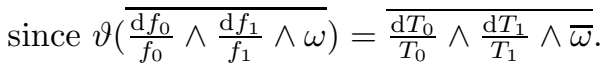


On the other hand, let $\omega \in \Omega_{A_{f_{0} f_{1}}}^{p}$ be a form with $\mathrm{d} \omega \in \Omega_{A_{f_{0}}}^{p}+\Omega_{A_{f_{1}}}^{p}$. Writing $\vartheta(\bar{\omega})$ in the form (31) and differentiating yields

$$
\begin{aligned}
\mathrm{d} \vartheta(\bar{\omega})= & \sum_{\mu, \nu \geq 1}\left(\left(\mathrm{~d} \alpha_{\mu \nu}+\mathrm{d} \beta_{\mu \nu} \wedge \mathrm{d} T_{0}+\mathrm{d} \gamma_{\mu \nu} \wedge \mathrm{d} T_{1}+\mathrm{d} \delta_{\mu \nu} \wedge \mathrm{d} T_{0} \wedge \mathrm{d} T_{1}\right) T_{0}^{-\mu} T_{1}^{-\nu}\right. \\
& -(-1)^{p} \mu\left(\alpha_{\mu \nu}+\gamma_{\mu \nu} \wedge \mathrm{d} T_{1}\right) T_{0}^{-\mu-1} T_{1}^{-\nu} \mathrm{d} T_{0} \\
& \left.-(-1)^{p} \nu\left(\alpha_{\mu \nu}+\beta_{\mu \nu} \wedge \mathrm{d} T_{0}\right) T_{0}^{-\mu} T_{1}^{-\nu-1} \mathrm{~d} T_{1}\right) \\
= & \sum_{\mu, \nu \geq 1} T_{0}^{-\mu} T_{1}^{-\nu}\left(\mathrm{d} \alpha_{\mu \nu}+\left(\mathrm{d} \beta_{\mu \nu}+(-1)^{p+1}(\mu-1) \alpha_{\mu-1, \nu}\right) \wedge \mathrm{d} T_{0}\right. \\
& +\left(\mathrm{d} \gamma_{\mu \nu}+(-1)^{p+1}(\nu-1) \alpha_{\mu, \nu-1}\right) \wedge \mathrm{d} T_{1} \\
& \left.+\left(\mathrm{d} \delta_{\mu \nu}+(-1)^{p}(\mu-1) \gamma_{\mu-1, \nu}+(-1)^{p+1}(\nu-1) \beta_{\mu, \nu-1}\right) \wedge \mathrm{d} T_{0} \wedge \mathrm{d} T_{1}\right) .
\end{aligned}
$$

Since in all terms of this expression both $T_{0}$ and $T_{1}$ have negative exponents and are contained in $\widehat{\Omega}_{B\left[\left[T_{0}, T_{1}\right]\right]_{T_{0}}}^{p}+\widehat{\Omega}_{B\left[\left[T_{0}, T_{1}\right]\right]_{T_{1}}}^{p}$, they must be zero in $\widehat{\Omega}_{B\left[\left[T_{0}, T_{1}\right]\right]_{T_{0} T_{1}}^{p}}$. Among others, this implies the relations

$$
\begin{gathered}
\mathrm{d} \beta_{\mu \nu}+(-1)^{p+1}(\mu-1) \alpha_{\mu-1, \nu}=0, \\
\mathrm{~d} \delta_{\mu \nu}+(-1)^{p}(\mu-1) \gamma_{\mu-1, \nu}+(-1)^{p+1}(\nu-1) \beta_{\mu, \nu-1}=0
\end{gathered}
$$

for all $\mu, \nu \geq 1$. In particular, (33) shows that $\delta_{1,1}$ is closed. We have to show that all other terms of $\omega$ can be integrated. To do so, define

$\eta:=\sum_{\mu \geq 2} \sum_{\nu \geq 1}(-1)^{p} \frac{T_{0}^{1-\mu}}{1-\mu} T_{1}^{-\nu}\left(-\beta_{\mu \nu}+\delta_{\mu \nu} \wedge \mathrm{d} T_{1}\right)+\sum_{\nu \geq 2}(-1)^{p+1} T_{0}^{-1} \frac{T_{1}^{1-\nu}}{1-\nu} \delta_{1, \nu} \wedge \mathrm{d} T_{0}$.

We check

$$
\begin{aligned}
\mathrm{d} \eta= & \sum_{\mu \geq 2} \sum_{\nu \geq 1}(-1)^{p}\left(\left(T_{0}^{-\mu} T_{1}^{-\nu} \mathrm{d} T_{0}-\frac{T_{0}^{1-\mu}}{1-\mu} \nu T_{1}^{-\nu-1} \mathrm{~d} T_{1}\right) \wedge\left(-\beta_{\mu \nu}+\delta_{\mu \nu} \wedge \mathrm{d} T_{1}\right)\right. \\
& \left.+\frac{T_{0}^{1-\mu}}{1-\mu} T_{1}^{-\nu}\left(-\mathrm{d} \beta_{\mu \nu}+\mathrm{d} \delta_{\mu \nu} \wedge \mathrm{d} T_{1}\right)\right) \\
& +\sum_{\nu \geq 2}(-1)^{p+1}\left(\left(-T_{0}^{-2} \frac{T_{1}^{1-\nu}}{1-\nu} \mathrm{d} T_{0}+T_{0}^{-1} T_{1}^{-\nu} \mathrm{d} T_{1}\right) \wedge \delta_{1, \nu} \wedge \mathrm{d} T_{0}\right. \\
& \left.+T_{0}^{-1} \frac{T_{1}^{1-\nu}}{1-\nu} \mathrm{d} \delta_{1, \nu} \wedge \mathrm{d} T_{0}\right) \\
= & \sum_{\mu \geq 2} \sum_{\nu \geq 1}\left((-1)^{p+1} \frac{T_{0}^{1-\mu}}{1-\mu} T_{1}^{-\nu} \mathrm{d} \beta_{\mu \nu}+T_{0}^{-\mu} T_{1}^{-\nu} \beta_{\mu \nu} \wedge \mathrm{d} T_{0}\right. \\
& \left.+\frac{T_{0}^{1-\mu}}{1-\mu}\left(-\nu T_{1}^{-\nu-1} \beta_{\mu \nu}+(-1)^{p} T_{1}^{-\nu} \mathrm{d} \delta_{\mu \nu}\right) \wedge \mathrm{d} T_{1}+T_{0}^{-\mu} T_{1}^{-\nu} \delta_{\mu \nu} \mathrm{d} T_{0} \wedge \mathrm{d} T_{1}\right) \\
& +\sum_{\nu \geq 2} T_{0}^{-1}\left(T_{1}^{-\nu} \delta_{1, \nu} \wedge \mathrm{d} T_{0} \wedge \mathrm{d} T_{1}+(-1)^{p+1} \frac{T_{1}^{1-\nu}}{1-\nu} \mathrm{d} \delta_{1, \nu} \wedge \mathrm{d} T_{0}\right) .
\end{aligned}
$$


Using (32) and (33) for $\mu=1$ we obtain

$$
\begin{aligned}
& \mathrm{d} \eta= \sum_{\mu \geq 2} \sum_{\nu \geq 1}\left(T_{0}^{1-\mu} T_{1}^{-\nu} \alpha_{\mu-1, \nu}+T_{0}^{-\mu} T_{1}^{-\nu} \beta_{\mu \nu} \wedge \mathrm{d} T_{0}\right. \\
&\left.+\frac{T_{0}^{1-\mu}}{1-\mu} T_{1}^{-\nu}\left(-(\nu-1) \beta_{\mu, \nu-1}+(-1)^{p} \mathrm{~d} \delta_{\mu \nu}\right) \wedge \mathrm{d} T_{1}\right) \\
&+\sum_{\substack{\mu, \nu \geq 1 \\
(\mu, \nu) \neq(1,1)}} T_{0}^{-\mu} T_{1}^{-\nu} \delta_{\mu \nu} \mathrm{d} T_{0} \wedge \mathrm{d} T_{1}+\sum_{\nu \geq 2} T_{0}^{-1} T_{1}^{1-\nu} \beta_{1, \nu-1} \wedge \mathrm{d} T_{0} \\
& \stackrel{(33)}{=} \sum_{\mu \geq 1} \sum_{\nu=1}^{s} T_{0}^{-\mu} T_{1}^{-\nu}\left(\alpha_{\mu, \nu}+\beta_{\mu \nu} \wedge \mathrm{d} T_{0}\right)+\sum_{\mu \geq 2} \sum_{\nu \geq 1} T_{0}^{1-\mu} T_{1}^{-\nu} \gamma_{\mu-1, \nu} \wedge \mathrm{d} T_{1} \\
& \quad+\sum_{\substack{\mu, \nu \geq 1 \\
(\mu, \nu) \neq(1,1)}} T_{0}^{-\mu} T_{1}^{-\nu} \delta_{\mu \nu} \mathrm{d} T_{0} \wedge \mathrm{d} T_{1} \\
&=\vartheta(\bar{\omega})-T_{0}^{-1} T_{1}^{-1} \delta_{1,1} \mathrm{~d} T_{0} \wedge \mathrm{d} T_{1},
\end{aligned}
$$

thus $\lambda \circ \operatorname{Res}([\bar{\omega}])=[\bar{\omega}]$.

In order to prove (17), we have to bound the degree of $\delta_{1,1}$ in (31). Note that by linearity it suffices to consider terms of the form

$$
\omega=\frac{a}{\left(f_{0} f_{1}\right)^{s}} \mathrm{~d} X_{i_{1}} \wedge \cdots \wedge \mathrm{d} X_{i_{p}}, \quad a \in A, 0 \leq i_{1}<\cdots<i_{p} \leq n, s \geq 1 .
$$

By Lemma 6.8 we have

$$
\begin{aligned}
b & :=\widehat{\psi}^{-1}(a)=\sum_{\mu, \nu \geq 0} b_{\mu \nu} T_{0}^{\mu} T_{1}^{\nu} \\
\Xi_{i} & :=\widehat{\psi}^{-1}\left(X_{i}\right)=\sum_{\mu, \nu \geq 0} b_{\mu \nu}^{(i)} T_{0}^{\mu} T_{1}^{\nu} \in B\left[\left[T_{0}, T_{1}\right]\right],
\end{aligned}
$$

where

$$
\operatorname{deg} b_{\mu \nu} \leq \gamma^{\mu+\nu} \operatorname{deg} a, \quad \operatorname{deg} b_{\mu \nu}^{(i)} \leq \gamma^{\mu+\nu} .
$$

It follows

$$
\vartheta(\bar{\omega})=\frac{b}{\left(T_{0} T_{1}\right)^{s}} \mathrm{~d} \Xi_{i_{1}} \wedge \cdots \wedge \mathrm{d} \Xi_{i_{p}}
$$

Moreover, we have

$$
\begin{aligned}
\mathrm{d} \Xi_{i} & =\sum_{\mu, \nu \geq 0}\left(\mathrm{~d} b_{\mu \nu}^{(i)} T_{0}^{\mu} T_{1}^{\nu}+b_{\mu \nu}^{(i)} \mu T_{0}^{\mu-1} T_{1}^{\nu} \mathrm{d} T_{0}+b_{\mu \nu}^{(i)} \nu T_{0}^{\mu} T_{1}^{\nu-1} \mathrm{~d} T_{1}\right) \\
& =\sum_{\mu, \nu \geq 0}\left(\mathrm{~d} b_{\mu \nu}^{(i)}+(\mu+1) b_{\mu+1, \nu}^{(i)} \mathrm{d} T_{0}+(\nu+1) b_{\mu, \nu+1}^{(i)} \mathrm{d} T_{1}\right) T_{0}^{\mu} T_{1}^{\nu} .
\end{aligned}
$$


The terms of $\vartheta(\bar{\omega})$ involving $d T_{0} \wedge d T_{1}$ are of the form

$$
\begin{aligned}
\pm\left(\mu_{1}+1\right)\left(\nu_{2}+1\right) b_{\mu \nu} b_{\mu_{1}+1, \nu_{1}}^{(i)} b_{\mu_{2}, \nu_{2}+1}^{(j)} & T_{0}^{\mu+\mu_{1}+\cdots+\mu_{p}-s} T_{1}^{\nu+\nu_{1}+\cdots+\nu_{p}-s} \\
\cdot & \mathrm{d} T_{0} \wedge \mathrm{d} T_{1} \wedge \mathrm{d}_{B} b_{\mu_{3}, \nu_{3}}^{\left(j_{1}\right)} \wedge \cdots \wedge \mathrm{d}_{B} b_{\mu_{p}, \nu_{p}}^{\left(j_{p-2}\right)}
\end{aligned}
$$

with some $0 \leq i, j, j_{1}, \ldots, j_{p-2} \leq n$ and $\mu, \nu, \mu_{1}, \nu_{1}, \ldots, \mu_{p}, \nu_{p} \geq 0$. To get the coefficient $\delta_{1,1}$ of $\mathrm{d} T_{0} / T_{0} \wedge \mathrm{d} T_{1} / T_{1}$, we have to consider the case $\mu+\mu_{1}+\cdots+\mu_{p}=$ $s-1$ and $\nu+\nu_{1}+\cdots+\nu_{p}=s-1$. Using that $\mathrm{d}_{B}$ is of degree 0 together with the estimate (34), it follows that $\delta_{1,1}$ is of degree

$$
\begin{aligned}
& \leq \operatorname{deg} b_{\mu \nu}+\operatorname{deg} b_{\mu_{1}+1, \nu_{1}}^{(i)}+\operatorname{deg} b_{\mu_{2}, \nu_{2}+1}^{(j)}+\operatorname{deg} b_{\mu_{3}, \nu_{3}}^{\left(j_{1}\right)}+\cdots+\operatorname{deg} b_{\mu_{p}, \nu_{p}}^{\left(j_{p-2}\right)} \\
& \leq \gamma^{\mu+\nu} \operatorname{deg} a+\gamma^{\mu_{1}+\nu_{1}+1}+\gamma^{\mu_{2}+\nu_{2}+1}+\gamma^{\mu_{3}+\nu_{3}}+\cdots+\gamma^{\mu_{p}+\nu_{p}} \\
& \leq \gamma^{2 s-1}(\operatorname{deg} a+p) .
\end{aligned}
$$

\section{Proof of the Main Theorem}

The effective Gysin sequence yields degree and order bounds for the de Rham cohomology of a smooth hypersurface.

Theorem 7.1. Let $f, g \in R=k\left[X_{1}, \ldots, X_{n}\right]$ with $d:=\operatorname{deg} f$ and $d^{\prime}:=\operatorname{deg} g$, such that $f$ is irreducible and $\frac{\partial f}{\partial X_{n}} \mid g$, and consider the smooth hypersurface $V:=\mathcal{Z}(f) \backslash \mathcal{Z}(g) \subseteq \mathbb{A}^{n}$. Then we have

$$
(p+2)\left(d+d^{\prime}+2\right)\left(2 d^{\prime}-d+3\right)^{2 p+3} \cdot(1,1) \in B\left(H_{\mathrm{dR}}^{p}(V)\right) .
$$

for all $p \in \mathbb{N}$.

Proof. Putting $f_{0}:=g X_{0}-1, f_{1}:=f$, we have the isomorphism

$$
V \stackrel{\simeq}{\longrightarrow} Z:=\mathcal{Z}\left(f_{0}, f_{1}\right) \subseteq \mathbb{A}^{n+1}, x \mapsto(1 / g(x), x),
$$

and the pull-back of differential forms shows

$$
\operatorname{deg}\left(H_{\mathrm{dR}}^{p}(Z)\right) \cdot(1,1) \in B\left(H_{\mathrm{dR}}^{p}(V)\right) .
$$

Thus, we have reduced to the setting of Theorem 6.3. Note that $d_{0}:=\operatorname{deg} f_{0}=$ $d^{\prime}+1$ and $d_{1}:=\operatorname{deg} f_{1}=d$. By Corollary 5.6 we have

$$
p \cdot(1,1) \in B\left(H^{p}\left(\frac{\Omega_{A_{f_{0} f_{1}}}^{\bullet}}{\Omega_{A_{f_{0}}}+\Omega_{A_{f_{1}}}^{\bullet}}\right)\right) .
$$

Theorem 6.3 implies

$\operatorname{deg}\left(H_{\mathrm{dR}}^{p-2}(Z)\right) \leq\left(2 d_{0}-d_{1}+1\right)^{2 p-1} p\left(d_{0}+d_{1}+1\right)=p\left(d+d^{\prime}+2\right)\left(2 d^{\prime}-d+3\right)^{2 p-1}$, which implies the claim. 
Proof of Theorem 1.1. Let $X \subseteq \mathbb{A}^{n}$ be a smooth closed variety. The zeroth cohomology is treated in $\S 3$, and the case $D=1$ is trivial, so we assume $n>$ $m \geq 1$ and $D \geq 2$.

First assume that $X$ is irreducible. Then, by Corollary 4.5 and Lemma 4.2 we can write $X=\bigcup_{i=0}^{m} U_{i}$, where $U_{i}=X \backslash \mathcal{Z}\left(g_{i}\right)$ is isomorphic to $V_{i}=Y_{i} \backslash \mathcal{Z}\left(g_{i}\right) \subseteq$ $\mathbb{A}^{m+1}$. Furthermore, in suitable coordinates $X_{1}, \ldots, X_{n}$ of $\mathbb{A}^{n}$, the isomorphism is given by the projection $\pi: U_{i} \rightarrow V_{i}$ onto the first $m+1$ coordinates, and we have $Y_{i}=\mathcal{Z}\left(f_{i}\right)$, where $f_{i} \in k\left[X_{1}, \ldots, X_{m+1}\right]$ is irreducible and monic in $X_{m+1}$, $g_{i}=\frac{\partial f_{i}}{\partial X_{m+1}}$, and $\operatorname{deg} f_{i} \leq D$. Now fix $1 \leq \ell \leq m$. For $p, q \in \mathbb{N}$ with $p+q=\ell$ consider a multi-index $0 \leq i_{0}<\cdots<i_{q} \leq m$. Set $i:=i_{0}, U^{\prime}:=U_{i_{0} \cdots i_{q}}$, and $V^{\prime}:=\pi\left(U^{\prime}\right)$. Note that $U^{\prime}=U_{i} \backslash \mathcal{Z}(h)$ with $h:=g_{i_{1}} \cdots g_{i_{q}}$. From Corollary 4.4 we obtain $H \in k\left[Y_{i}\right]$ with $\operatorname{deg} H \leq D \operatorname{deg} h$ such that $k\left[V^{\prime}\right]=k\left[Y_{i}\right]_{g_{i} H}$. We can assume $d:=\operatorname{deg} f_{i} \geq 2$, and with $g:=g_{i} H$ we have $d-1 \leq d^{\prime}:=\operatorname{deg} g \leq$ $d-1+\operatorname{deg} H \leq(D-1)(q D+1) \leq \ell D^{2}+D-2$. Theorem 7.1 implies

$$
(p+2)\left(d+d^{\prime}+2\right)\left(2 d^{\prime}-d+3\right)^{2 p+3} \cdot(1,1) \in B\left(H_{\mathrm{dR}}^{p}\left(V_{i}\right)\right) .
$$

Moreover,

$$
\begin{aligned}
(p+2) & \left(d+d^{\prime}+2\right)\left(2 d^{\prime}-d+3\right)^{2 p+3} \\
& \leq(p+2)(D+(D-1)(q D+1))(2(D-1)(q D+1)+1)^{2 p+3} \\
& \leq(p+2)\left(\ell D^{2}+2 D\right)(2 D(q(D-1)+1))^{2 p+3} \\
& \leq 6 \ell^{2} D^{2}\left(2 \ell D^{2}\right)^{2 \ell+3}=3 \cdot 2^{2 \ell+4} \ell^{2 \ell+5} D^{4 \ell+8} .
\end{aligned}
$$

Using Corollary 4.4 we conclude

$$
(s, d):=3 \cdot 2^{2 \ell+4} \ell^{2 \ell+5} D^{4 \ell+8} \cdot(\ell D, 1) \in B\left(H_{\mathrm{dR}}^{p}\left(U_{i_{0} \cdots i_{q}}\right)\right),
$$

Since this holds for all $p+q=\ell$ and all $i_{0}<\cdots<i_{q}$, Theorem 5.3 shows that

$$
\begin{aligned}
\operatorname{deg} H_{\mathrm{dR}}^{\ell}(X) & \leq d+2 D(\ell+1)(s+\ell)^{m}(D-1)^{m} \\
& \leq 2 D(\ell+1)(\ell D d+\ell)^{m} D^{m} \\
& \leq 4 \ell^{m+1}(D d+1)^{m} D^{m+1} \\
& \stackrel{(35)}{\leq} 4 \ell^{m+1}\left(2^{2 \ell+6} \ell^{2 \ell+5} D^{4 \ell+9}\right)^{m} D^{m+1} \\
& =2^{2 \ell m+6 m+2} \ell^{2 \ell m+6 m+1} D^{4 \ell m+10 m+1} .
\end{aligned}
$$

Finally, for reducible $X$, Corollary 4.1 implies the claim.

\section{Acknowledgements}

The author thanks Sergei Yakovenko for asking the question addressed in this paper and bringing to his attention the solution of the infinitesimal Hilbert 16th problem [5]. He also thanks Cornelia Rottner for finding some inaccuracies in a previous version. Example 2.3 is due to her. He is also grateful to the Hausdorff Center for Mathematics, Bonn, as well as the Lucerne University of Applied Sciences and Arts for their kind support. 


\section{References}

[1] S. Basu. Computing the first few Betti numbers of semi-algebraic sets in single exponential time. J. Symbolic Comput., 41(10):1125-1154, 2006.

[2] S. Basu. Algorithmic semi-algebraic geometry and topology - recent progress and open problems. In Surveys on discrete and computational geometry, volume 453 of Contemp. Math., pages 139-212. Amer. Math. Soc., Providence, RI, 2008.

[3] S. Basu, R. Pollack, and M.-F. Roy. Algorithms in Real Algebraic Geometry, volume 10 of Algorithms and Computation in Mathematics. Springer Verlag, Berlin Heidelberg New York, 2003.

[4] E. Bierstone, D. Grigoriev, P. Milman, and J. Wlodarczyk. Effective Hironaka resolution and its complexity. Asian J. Math., 15(2):193-228, 2011.

[5] G. Binyamini, D. Novikov, and S. Yakovenko. On the number of zeros of abelian integrals. Invent. Math., 181:227-289, 2010.

[6] W.D. Brownawell. Bounds for the degrees in the Nullstellensatz. Ann. of Math. (2), 126(3):577-591, 1987.

[7] P. Bürgisser and P. Scheiblechner. On the complexity of counting components of algebraic varieties. J. Symb. Comp., 44(9):1114-1136, 2009. Effective Methods in Algebraic Geometry.

[8] L. Caniglia, A. Galligo, and J. Heintz. Equations for the projective closure and effective Nullstellensatz. Discr. Appl. Math., 33(1-3):11-23, 1991.

[9] P. Deligne and A. Dimca. Filtrations de Hodge et par l'ordre du pôle pour les hypersurfaces singulières. Annales scientifiques de l'École Normale Supérieure, 23(4):645-656, 1990.

[10] A. Dimca. On the Milnor fibrations of weighted homogeneous polynomials. Compositio Mathematica, 76(1-2):19-47, 1990.

[11] A. Dimca. On the de Rham cohomology of a hypersurface complement. Amer. J. Math., 113(4):763-771, 1991.

[12] A. Dimca. Singularities and Topology of Hypersurfaces. Universitext. Springer Verlag, 1992.

[13] D. Eisenbud. Commutative Algebra with a View Toward Algebraic Geometry, volume 150 of Graduate Texts in Mathematics. Springer-Verlag, New York, 1995.

[14] M. Giusti, J. Heintz, J.E. Morais, and L.M. Pardo. When polynomial equation systems can be solved fast? In G. Cohen, M. Giusti, and T. Mora, editors, Applied Algebra, Algebraic Algorithms, and Error Correcting Codes (AAECC-11), volume 948 of Lecture Notes in Computer Science, pages 205-231. Springer, Berlin, 1995. 
[15] M. Giusti, G. Lecerf, and B. Salvy. A Gröbner free alternative for polynomial system solving. J. Compl., 17(1):154-211, 2001.

[16] P. Griffiths. On the periods of certain rational integrals: I. Annals of Mathematics. Second Series, 90(3):460-495, 1969.

[17] A. Grothendieck. Éléments de géométrie algébrique. IV. Étude locale des schémas et des morphismes de schémas. I. Inst. Hautes Études Sci. Publ. Math., (20):259, 1964.

[18] A. Grothendieck. On the de Rham cohomology of algebraic varieties. Publications Mathématiques IHES, 39:93-103, 1966.

[19] R. Hartshorne. Local cohomology. A seminar given by A. Grothendieck, Harvard University, Fall. Lecture Notes in Mathematics, Vol. 41. SpringerVerlag, Berlin, 1967.

[20] R. Hartshorne. Ample subvarieties of algebraic varieties. Notes written in collaboration with C. Musili. Lecture Notes in Mathematics, Vol. 156. Springer-Verlag, Berlin, 1970.

[21] R. Hartshorne. On the de Rham cohomology of algebraic varieties. Publications Mathématiques de L'IHÉS, 45:6-99, 1975. 10.1007/BF02684298.

[22] R. Hartshorne. Algebraic geometry. Springer-Verlag, New York, 1977.

[23] J. Heintz. Definability and fast quantifier elimination in algebraically closed fields. Theoret. Comp. Sci., 24:239-277, 1983.

[24] Z. Jelonek. On the effective Nullstellensatz. Invent. Math., 162(1):1-17, 2005 .

[25] J. Kollár. Sharp effective Nullstellensatz. J. Amer. Math. Soc., 1(4):963975, 1988.

[26] J. Kollár. Effective Nullstellensatz for arbitrary ideals. J. Eur. Math. Soc., 1(3):313-337, 1998.

[27] E. Kunz. Kähler Differentials. Advanced Lectures in Mathematics. Vieweg, Wiesbaden, 1986.

[28] J. McCleary. A User's Guide to Spectral Sequences, volume 12 of Mathematics Lecture Series. Publish or Perish, Inc, Wilmington, Delaware, 1985.

[29] D. Mumford. Algebraic Geometry I: Complex Projective Varieties, volume 221 of Grundlehren der mathematischen Wissenschaften. Springer-Verlag, Berlin Heidelberg New York, 1976.

[30] T. Oaku and N. Takayama. An algorithm for de Rham cohomology groups of the complement of an affine variety via D-module computation. Journal of Pure and Applied Algebra, 139:201-233, 1999. 
[31] L.M. Pardo. How lower and upper complexity bounds meet in elimination theory. In G. Cohen, M. Giusti, and T. Mora, editors, Applied Algebra, Algebraic Algorithms, and Error Correcting Codes (AAECC-11), volume 948 of Lecture Notes in Computer Science, pages 33-69. Springer, Berlin, 1995.

[32] J. Sabia and P. Solernó. Bounds for traces in complete intersections and degrees in the Nullstellensatz. Applicable Algebra in Engineering, Communication and Computing, 6:353-376, 1995.

[33] P. Scheiblechner. On the complexity of deciding connectedness and computing Betti numbers of a complex algebraic variety. J. Compl., 23(3):359-379, 2007.

[34] P. Scheiblechner. On a generalization of Stickelberger's theorem. J. Symb. Comp., 45(12):1459 - 1470, 2010. MEGA'2009.

[35] P. Scheiblechner. Castelnuovo-Mumford regularity and computing the de Rham cohomology of smooth projective varieties. arXiv:0905.2212v3, to appear in Foundations of Computational Mathematics, 2012.

[36] P. Scheiblechner. Effective de Rham cohomology - the hypersurface case. arXiv:1112.2489v1, to appear in the Proceedings of ISSAC 2012, 2012.

[37] U. Walther. Algorithmic computation of de Rham cohomology of complements of complex affine varieties. J. Symb. Comp., 29(4-5):795-839, 2000.

[38] U. Walther. Algorithmic determination of the rational cohomology of complex varieties via differential forms. In Symbolic computation: solving equations in algebra, geometry, and engineering (South Hadley, MA, 2000), volume 286 of Contemp. Math., pages 185-206. Amer. Math. Soc., Providence, RI, 2001. 